\title{
Spatiotemporal regularization for digital image correlation: Application to infrared camera frames
}

\author{
A. Charbal ${ }^{* 1,2}$, S. Roux ${ }^{\dagger 1}$, F. Hild ${ }^{\ddagger 1}$, and L. Vincent ${ }^{\S 2}$ \\ ${ }^{1}$ LMT, ENS Paris-Saclay / CNRS / University Paris-Saclay \\ 61 avenue du Président Wilson, 94235 Cachan cedex, France \\ ${ }^{2}$ DEN-Service de Recherches Métallurgiques Appliquées, \\ CEA, University Paris-Saclay, 91191 Gif-sur-Yvette, France
}

January 29, 2018

\begin{abstract}
The spatiotemporal response of a stainless steel plate undergoing cyclic laser shock is recorded with an infrared camera, and Digital Image Correlation (DIC) is used to analyze both displacement and temperature fields. Two very challenging difficulties are addressed: i) large gray level variations (due to temperature changes) and ii) convection effects affecting images. To this aim, a spatiotemporal regularization is designed exploiting a numerical model of the test. The thermomechanical space-time predictions are first processed through Karhunen-Loève decomposition to extract dominant temporal and spatial modes. The temporal modes are then inserted in a spatiotemporal DIC framework to estimate the experimental spatial modes that account for both gray level variations (and hence temperature) and displacement fields. It is shown that with only three modes, the full thermomechanical response of the material is captured. The temporal regularization issued from the model also allows the spurious effect of convection to be filtered out. Because of the drastic decrease in the number of degrees of freedom due to data reduction, the number of analyzed frames can be reduced from 50 down to 6 to capture the thermomechanical response, thereby leading to an enhanced efficiency.
\end{abstract}

\footnotetext{
*alc517@lehigh.edu - Present address: Lehigh Univ., Packard Lab, 19 Memorial D. West, Bethlehem, PA 18015, USA

†stephane.roux@ens-paris-saclay.fr

${ }^{\ddagger}$ hild@ens-paris-saclay.fr

${ }^{\S}$ ludovic.vincent@cea.fr
} 


\section{Introduction}

Digital Image Correlation (DIC) applied to experimental mechanics appeared in the early 1980's [1-4]. The large amount of information provided by such techniques allows the understanding of mechanical response of materials to be enhanced through various experimental tests [5. DIC generally consists in registering two images recorded at different loading stages assuming brightness conservation. The displacement fields between reference and deformed states can be measured. Different DIC methodologies have been proposed over the years $2,6[9]$. Local approaches for instance decompose the images into small interrogation windows (or "zones of interest", ZOIs) and cross-correlation between corresponding ZOIs in reference and deformed images provides the displacement of each ZOI center [8].

To control the intrinsic ill-posedness of the DIC problem when a fine spatial resolution is sought, and its resulting noise sensitivity, different strategies have been proposed to provide regularization (through a reduction in the number of degrees of freedom) and yet enough flexibility not to betray the actual kinematics. Global DIC methods exploiting a mesh with finite element (FE) formulation 10 easily endow the displacement fields with continuity. Higher order finite-elements [11] or other parameterizations (spline [12, NURBS 13]) provide higher regularity and better conditioning of the DIC problem. For those variants the whole region of interest (ROI) is to be considered at once, hence the term "global" 7 .

Rather than choosing a specific space in which displacement fields are sought (i.e., "hard regularization" as above), it is also possible to turn to "soft regularization," in the spirit of Tikhonov [14], and penalize irregular or unexpected displacements. For DIC, specific mechanical filters have been proposed [15, 16]. When, in addition to accounting for a displacement field, gray level corrections are necessary, say to register infrared (IR) frames, a (even) more general approach can be implemented [17, 18]. The same type of filtering can be applied to dampen out inherent spatial fluctuations [19].

As regularization is enhanced, the danger is to betray reality. Thus, one safe way to proceed is to incorporate a model that is as faithful as possible to the actual case. Such approaches are called Integrated-DIC (I-DIC) and reduce the number of unknowns to only few parameters, which describe a defined kinematics. The constrained registration is then limited to the sensitivity fields (i.e., variation of the displacement field with respect to unknown parameters of the model) provided by either closed-form solutions [20,21] or FE predictions 22,26$]$.

All those regularization methods address DIC between a single pair of images but many experimental applications necessitate a full movie or sequence of images and the spatiotemporal information is of interest. Along the previous lines, it is also possible to include the time dimension in the DIC formalism. A global route has already been proposed [27, 28 where time integration over a series of frames allows a 3D problem (with 2D spatial coordinates plus time) to be analyzed with the DIC algorithm and revealed useful to analyze various experimental cases $27-29]$. An I-DIC procedure including time has also been 
proposed [24, 25, 30] in order to calibrate various material parameters.

In the present study, it is proposed to incorporate a spatiotemporal regularization, which is constructed from an FE model of the experiment. Model reduction is used in order to define temporal modes from the model, and those modes are inserted in a spatiotemporal DIC framework. The algorithm is applied to the case of laser shocks on a stainless steel, which was already investigated with an incremental DIC routine 31. When no temporal constraint is introduced, temporal fluctuations are observed. They are due to small displacement amplitudes that are measured, which are highly sensitive to experimental noise. In order to assess the robustness of this new technique, two challenging situations are considered. First high gray level (GL) variations are studied. The second sequence deals with thermal convection.

A general introduction to DIC, where the notations are defined in a general space-time framework including GL corrections, is presented in Section 2. The formalism of global DIC is briefly discussed in order to set the basic equations needed to introduced temporal regularization. Then it is proposed to use a model reduction technique from a numerical thermomechanical modeling of the studied case to provide the needed temporal modes. Section 3 shows that the proposed technique allows us to cope with a very challenging case of gray level changes due to a laser heating. The same experimental set-up is also used to provide a second challenging case study where convection introduces a "ghost motion." As the latter follows a time evolution that is different from the retained modes, the temporal regularization provides a remarkable robustness to such very detrimental artifacts. The large reduction in the degrees of freedom allows the question of saving on the number of studied time frames to be addressed, a process called "pruning," and studied in Section 4. It is shown that the number of images can be cut down by more than a factor of 10 without much impact on the determined solution, which is now interpolated in time over the entire period and not just at the studied instants.

\section{Spatiotemporal regularization of DIC}

The space/time DIC approach follows the same general principles of Ref. [28] while the algorithm is implemented in the same framework as Ref. [16]. The use of unstructured T3 mesh support and GL conservation assumption relaxation allows us to handle images with GL variations [19]. In the region of interest (ROI) dedicated spatial regularization kernels are added to the DIC cost function. Such filters penalize displacement field fluctuations [16] and GL correction fields [19]. Regularization over the time coordinate can be applied by integrating over few frames 29] or series of frames [28 to filter out unwanted time fluctuations. However in this work another route is preferred and uses a model-based representation, which is a model reduced using Karhunen-Loève decomposition (or KLD 32,33]) to regularize the time evolution. Such choice also allows an optimized search of the sought fields to be constructed. 


\subsection{Generalized optical flow}

The acquired movie consists of GL-valued scalar fields denoted $f(\boldsymbol{x}, t)$ where $\boldsymbol{x}$ stands for the pixel coordinates and $t$ for the time or frame number. The reference state is considered to be acquired when no loading is applied and corresponds to the first frame of the movie, $f_{0}(\boldsymbol{x})=f(\boldsymbol{x}, t=0)$. The subsequent frames are assumed to contain the same GL information advected by the surface motions $\boldsymbol{u}(\boldsymbol{x}, t)$ generated by the experiment. The gray level conservation, which is very often considered in standard DIC algorithms is expressed as

$$
f(\boldsymbol{x}+\boldsymbol{u}(\boldsymbol{x}, t), t)=f_{0}(\boldsymbol{x})
$$

When IR frames are exploited [17, 18 for which the temperature field varies, the GL conservation assumption is not satisfied and other formulations $66,8,34,35$ are necessary. The following extension will be used herein

$$
f_{0}(\boldsymbol{x})=a(\boldsymbol{x}, t)+(1+b(\boldsymbol{x}, t)) f(\boldsymbol{x}+\boldsymbol{u}(\boldsymbol{x}, t), t)
$$

where displacement $\boldsymbol{u}(\boldsymbol{x}, t)$, brightness $a(\boldsymbol{x}, t)$ and contrast $b(\boldsymbol{x}, t)$ are space/time fields.

The minimization of the quadratic norm of the residual defined as the difference between left and right members of Equation (2) reads

$$
\Phi_{c}^{2}=\sum_{t \in \tau} \sum_{\boldsymbol{x} \in R O I}\left(f_{0}(\boldsymbol{x})-a(\boldsymbol{x}, t)-(1+b(\boldsymbol{x}, t)) f(\boldsymbol{x}+\boldsymbol{u}(\boldsymbol{x}, t), t)\right)^{2}
$$

where $\tau$ and $R O I$ are the considered time and spatial domains. All the unknown fields are gathered in the vector $\boldsymbol{v}(\boldsymbol{x}, t) \equiv\left\{u_{x}(\boldsymbol{x}, t), u_{y}(\boldsymbol{x}, t), a(\boldsymbol{x}, t), b(\boldsymbol{x}, t)\right\}$. The minimization of the non linear DIC problem in Equation (3) is performed through a Gauss-Newton scheme [7]. It is convenient to introduce the corrected image series, $\widetilde{f}(\boldsymbol{x}, t)$, which depends on the current determination $\tilde{v}(\boldsymbol{x}, t)$ of $\boldsymbol{v}(\boldsymbol{x}, t)$, such that

$$
\widetilde{f}(\boldsymbol{x}, t)=\tilde{a}(\boldsymbol{x}, t)+(1+\tilde{b}(\boldsymbol{x}, t)) f(\boldsymbol{x}+\tilde{u}(\boldsymbol{x}, t), t)
$$

For each iteration, the current space/time gray level residual reads

$$
\rho(\boldsymbol{x}, t)=\sum_{t \in \tau}\left(f_{0}(\boldsymbol{x})-\widetilde{f}(\boldsymbol{x}, t)\right)
$$

The correction to each unknown, $\delta \boldsymbol{v}(\boldsymbol{x}, t)$, is computed iteratively by solving the linearized DIC problem

$$
\boldsymbol{M}(\boldsymbol{x}, t) \delta \boldsymbol{v}(\boldsymbol{x}, t)=\boldsymbol{r}(\boldsymbol{x}, t)
$$

The expression of the DIC matrix $\boldsymbol{M}(\boldsymbol{x}, t)$ and right hand side vector $\boldsymbol{r}(\boldsymbol{x}, t)$ use the sensitivity fields

$$
s^{i}(\boldsymbol{x}, t) \equiv \frac{\partial \widetilde{f}(\boldsymbol{x}, t)}{\partial v^{i}(\boldsymbol{x}, t)} \approx\left\{f_{0, x}, f_{0, y}, 1, f\right\}(\boldsymbol{x}, t)
$$


so that

$$
\boldsymbol{M}(\boldsymbol{x}, t)=\boldsymbol{s}(\boldsymbol{x}, t)^{\top} \boldsymbol{s}(\boldsymbol{x}, t)
$$

and

$$
\boldsymbol{r}(\boldsymbol{x}, t)=\boldsymbol{s}(\boldsymbol{x}, t)^{\top} \rho(\boldsymbol{x}, t)
$$

$\boldsymbol{M}$ and $\boldsymbol{r}$ are defined pixel- and time-wise for all unknown fields. However the problem is ill-posed as the rank of $\boldsymbol{M}$ is less than the number of unknowns.

To circumvent this so-called aperture issue, an FE decomposition $7,10,17$ is exploited and will restore well-posedness and better condition the DIC problem. In this framework, $\boldsymbol{v}(\boldsymbol{x}, t)$ is decomposed over elementary functions $\boldsymbol{\xi}_{i}(\boldsymbol{x}, t)$ the dyadic product of nodal shape functions defined over a spatial mesh, $\boldsymbol{\chi}_{i}$ and time functions $\psi_{j}(t)$

$$
\begin{aligned}
\boldsymbol{v}(\boldsymbol{x}, t) & =\sum_{i} \boldsymbol{\nu}_{i} \boldsymbol{\xi}_{i}(\boldsymbol{x}, t) \\
& =\sum_{j, k} \nu_{j k} \boldsymbol{\chi}_{j}(\boldsymbol{x}) \psi_{k}(t)
\end{aligned}
$$

where $\{\boldsymbol{\nu}\}$ is the column vector gathering all unknown amplitudes $\nu_{j k}$. Exploiting the fact that $\boldsymbol{M}$ is independent of time, the vector gathering the incremental corrections to all the unknowns $\{\delta \boldsymbol{\nu}\}$ is solution to

$$
\left[\boldsymbol{M}_{D I C}\right]\{\delta \boldsymbol{\nu}\}=\left\{\boldsymbol{r}_{D I C}\right\}
$$

where

$$
\left[\boldsymbol{M}_{D I C}\right]=\sum_{t \in \tau} \sum_{\boldsymbol{x} \in R O I} \boldsymbol{\xi}^{\top}(\boldsymbol{x}, t) \boldsymbol{M}(\boldsymbol{x}, t) \boldsymbol{\chi}(\boldsymbol{x}, t)
$$

and

$$
\left\{\boldsymbol{r}^{M}\right\}=\sum_{t \in \tau} \sum_{\boldsymbol{x} \in R O I} \boldsymbol{\xi}^{\top}(\boldsymbol{x}, t) \boldsymbol{r}(\boldsymbol{x}, t)
$$

The displacement and gray level changes $\{\boldsymbol{\nu}\}$ are updated iteratively by $\{\delta \boldsymbol{\nu}\}$ until the increment is less than a prescribed threshold (i.e., $10^{-5}$ in the sequel). The mesh and time discretizations act as spatiotemporal regularization [28]. One possible route consists in considering a priori knowledge of the time evolution, which will dictate the expression of $\psi_{k}(t)$. Let us first discuss the soft spatial regularization, which is similar to that introduced in Refs. [15, 16].

\subsection{Soft spatial regularization}

The interested reader is referred to Refs. 15, 16] for technical details. Let us simply mention that steep variations of $\boldsymbol{v}$ in space are penalized by the quadratic norm of a (discretized) second order differential operator in the bulk of the domain (i.e., inner nodes). Issued from the equilibrium gap for the displacement fields and Laplacian gap for the brightness corrections, a quadratic form

$$
\boldsymbol{\Phi}_{e}^{2}=(1 / 2)\{\boldsymbol{\nu}\}^{\top}[\boldsymbol{R}]\{\boldsymbol{\nu}\}
$$

weighted by $\omega$ is added to $\Phi_{c}^{2}$, to form the total cost function $\Phi_{t}^{2}=\Phi_{c}^{2}+\omega \Phi_{e}^{2}$ to be minimized. It can be shown that $\omega$ is proportional to the fourth power of a regularization length scale, $\ell$, that can be adjusted at will. 


\subsection{Temporal regularization}

Up to now, the mesh and temporal shape functions are arbitrary. When the mesh size is smaller than the regularization length, the mesh discretization plays no role anymore, and hence only a scalar length (i.e., weight) is used to continuously adjust the fineness of the spatial degrees of freedom (or spatial resolution). Time decomposition is proposed in the following to result from a modeling of the problem of interest.

The heat transfer problem is first solved to provide the temperature field $T(\boldsymbol{x}, t)$. Then the 3D temperature field $T(\boldsymbol{x}, t)$ is prescribed to the sample volume in order to solve the thermomechanical problem, and compute the displacement field $\boldsymbol{u}(\boldsymbol{x}, t)$. Model-reduction techniques are then used to reduce the number of effective degrees of freedom. The same concepts exist in different forms ranging from Karhunen-Loève Decomposition (KLD), Singular Value Decomposition (SVD), Principal Component Approximation (PCA) to Proper Orthogonal Decomposition (POD). The idea is to approximate the temperature field (or displacement alike) as

$$
T(\boldsymbol{x}, t) \approx \sum_{k=1}^{N_{\text {mode }}} p_{k}^{T}(\boldsymbol{x}) h_{k}^{T}(t)
$$

where $p_{k}^{T}(\boldsymbol{x})$ and $h_{k}^{T}(t)$ are respectively the $k^{\text {th }}$ spatial and temporal mode. By increasing the number of modes $N_{\text {mode }}$, this approximation can be made arbitrarily accurate, but the key idea is rather to reduce $N_{\text {mode }}$ to a low value, and often, only few modes are sufficient to provide a fair approximation to $T$ and similarly for $\boldsymbol{u}$.

The temporal modes $h_{k}^{T}(t)$ resulting from the KLD of the temperature field are natural candidates to use as $\psi_{k}(t)$ functions. Likewise, the temporal modes $h_{k}^{u}(t)$ coming from the displacement fields $\boldsymbol{u}(\boldsymbol{x}, t)$ are possible choices for $\psi_{k}(t)$ functions when displacement modes are considered. Thus the brightness correction is decomposed as

$$
a(\boldsymbol{x}, t)=\sum_{i k} a_{i k} \boldsymbol{\chi}_{i}(\boldsymbol{x}) h_{k}^{T}(t)
$$

and similarly for the contrast $b$ field. Displacements are decomposed over a different basis in time

$$
\boldsymbol{u}(\boldsymbol{x}, t)=\sum_{i k} \boldsymbol{u}_{i k} \boldsymbol{\chi}_{i}(\boldsymbol{x}) h_{k}^{u}(t)
$$

This decomposition allows the corresponding gray level spatial modes $p_{k}^{T}(\boldsymbol{x})=$ $\sum_{i} a_{i k} \boldsymbol{\chi}_{i}(\boldsymbol{x})$ and displacement modes $p_{k}^{u}(\boldsymbol{x})=\sum_{i} \boldsymbol{u}_{i k} \boldsymbol{\chi}_{i}(\boldsymbol{x})$ to be constructed.

Let us note that such approach allows for memory space saving (i.e., only three spatial and temporal modes for each unknown fields are required to describe the full history in comparison with one field for each sought parameter in image-per-image approaches). Moreover, each mode can be treated independently (however the image correction step to be performed on each image of the series is the most time consuming part). 
In the following the number of modes that will be considered either for the temperature or the displacement fields is 3 , i.e., a very small number that will trigger a rather efficient regularization, and yet a good fidelity to the physical problem at hand.

\section{Experimental cases: laser shock assessment}

\subsection{Experimental setup}

In order to better understand thermal fatigue of stainless steel plates (label (1) in Figure 1(a)), a new experimental setup has been proposed [36]. The sample is a polished plate of dimensions $270 \times 40 \times 7 \mathrm{~mm}^{3}$. A natural preoxidation of the surface of interest provides the marking needed for DIC both in the visible and IR wavelength domains. Two copper clamps (label (2) in Figure 1(a)) allow the sample to be heated up to a starting temperature of $400{ }^{\circ} \mathrm{C}$ by Joule effect. The mean temperature of the sample is controlled by a type $\mathrm{K}$ thermocouple implanted in the center of the sample (mid-thickness, length and width) The thermal fatigue test is performed in helium atmosphere (after preliminary secondary vacuum is applied). In order to maintain the thermal fatigue machine walls at room temperature, helium is cooled thanks to a closed circuit, and forced convection is achieved within the chamber. A pulsed laser (TruPulse 156 manufactured by Trumpf, $\lambda=1064 \mathrm{~nm}$, labelled (3) in Figure 1(b)), is used to induce cyclic thermal shocks in the center of the sample surface. The thermal shock frequency is $1 \mathrm{~Hz}$ with a pulse duration of $50 \mathrm{~ms}$. The laser is inclined, relative to the sample surface, by an angle of $30^{\circ}$, which explains why the temperature distribution is elliptical and not circular.

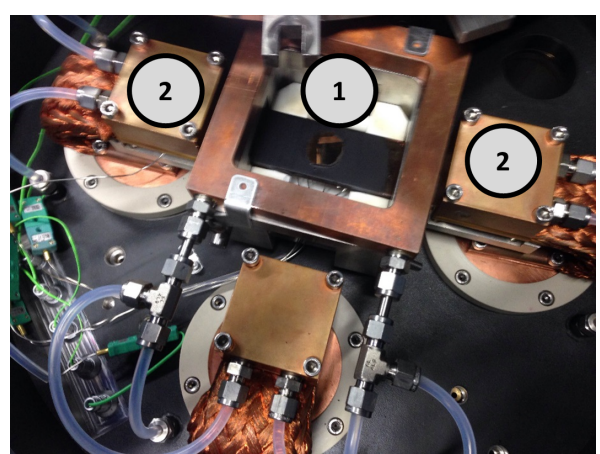

(a)

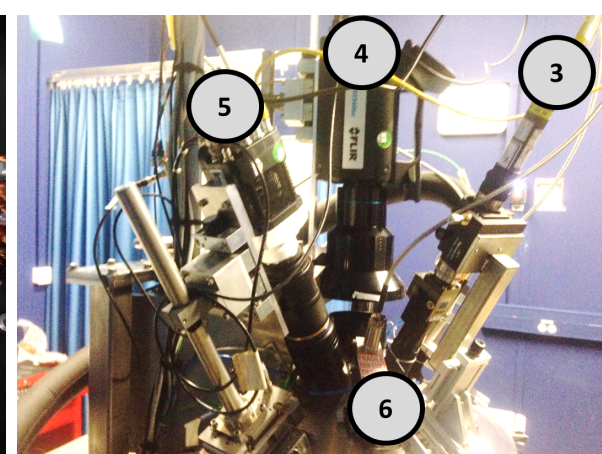

(b)

Figure 1: (a) Stainless steel sample (1), held by two copper clamps (2), onto which thermal shocks are applied. (b) Experimental setup with laser source (3) , IR (4) and visible light (5) cameras, and pyrometer (6)

Two experimental cases will be presented:

- IR frames are acquired with an x6540sc FLIR camera whose definition is $640 \times 512$ pixels and spectral range $\lambda=3970-4010 \mathrm{~nm}$ (label (4) in 
Figure 1(b)) with a $\times 1$ magnification lens (pitch $=15 \mu \mathrm{m})$. The displacement and temperature fields can be measured simultaneously with an acquisition frequency of $100 \mathrm{~Hz}$. For this particular case, the laser power density is set to reach an amplitude of temperature variation equal to $210{ }^{\circ} \mathrm{C}$.

IR image correlation (IRIC) is performed by considering reference and deformed images recorded within a same laser pulse. The cycle lasts 1 second as the thermal shock frequency is set to $1 \mathrm{~Hz}$. As a result no oxidation effects are observed on the images from the beginning (reference state) to the end of the pulse (various deformed states). In order to estimate the "true" temperature, the mean ${ }^{1}$ emissivity is determined by applying consecutive constant thermal steps (controlled by thermocouple) prior to the actual test. The sample was heated up by Joule effect using the copper clamps. These verifications have shown that no variations of emissivity with the applied temperature can be detected [37.

The ROI is selected to be as large as possible. For IR frames, the coordinates of the top left corner of the ROI are $x=30$ pixels and $y=30$ pixels, and the bottom right corner of the ROI is $x=495$ pixels and $y=620$ pixels. The considered spatial mesh size for the DIC calculation is 10 pixels, and the time discretization corresponds to a fine mesh of 20 frames during $50 \mathrm{~ms}$ (laser pulse duration) and 30 frames lasting $0.95 \mathrm{~s}$ or during the cooling regime. The time discretization is following that selected in the FE analysis but one can use interpolation functions and exploit more frames. For instance the use of a stroboscopic reconstruction allows 600 frames to be captured during one cycle [31, 36]. However in Section 4 it will be shown that the number of frames can be drastically reduced and still yield good results.

- In the second case, helium circulation is kept on while acquiring to generate a perturbation and the images are used as a proof of concept for the proposed methodology. The images acquired by the visible light camera (label (6) in Figure 1(b)) equipped with a $\times 1$ magnification lens (pitch $=10 \mu \mathrm{m}$ ) are exploited as the angle of inclination increases the sensitivity of the optical tools to the forced convection effect. The inhomogeneous distribution of hot/cold helium in the chamber perturbs the light path through the temperature dependence of the refraction index. This phenomenon produces apparent (but artifactual) motions when analyzed by DIC. The numerical model of this second case shows that, in spite of a lower temperature amplitude of $150{ }^{\circ} \mathrm{C}$, the temporal evolution is similar as in the first case, and hence the same temporal modes will be used.

\footnotetext{
${ }^{1}$ In the present case the mean emissivity of the surface was actually determined. The speckles, which were generated by pre-oxidation, provide a nonuniform emissivity distribution. This is necessary to provide enough contrast for DIC (IRIC) measurement purposes. The difference between the highest and lowest emissivity levels did not exceed 0.1. Using the mean emissivity then leads to slight under- or overestimates locally. However, because of the larger scales involved in the thermal loading (i.e., variation of temperature generated by the laser), this homogenized emissivity assumption leads to negligible effects in the sequel.
} 
In both cases, an IR pyrometer (label (6) in Figure 1(b)) allows the thermal loading to be monitored in real-time. Both cameras are synchronized and triggered with the laser signal [31,36]. These acquisitions can be combined for so-called hybrid stereocorrelation [38]. However, in the following, only data sets from one camera will be discussed for the two considered test cases.

A pair of IR reference and deformed images is displayed in Figure 2, In the right image, the effect of laser heating on the stainless steel sample (namely temperature elevation) is prominent. Such thermal response generates, in the IR spectral range, a large change of the emitted radiative flux toward the camera sensor. As a result, the deformed image undergoes a large gray level variation in the impacted zone in addition to the gray level advection that is caused by the thermomechanical response of the sample. Without considering additional gray level corrections in the DIC formalism such images cannot be registered.

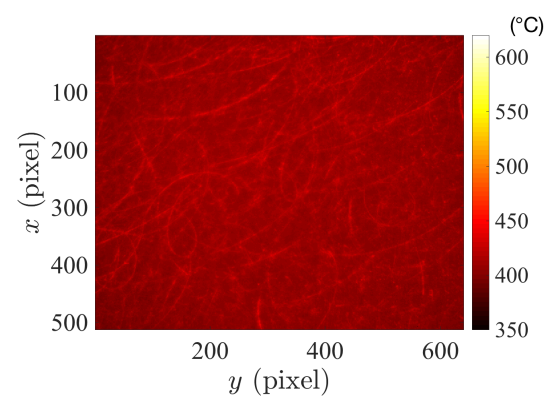

(a)

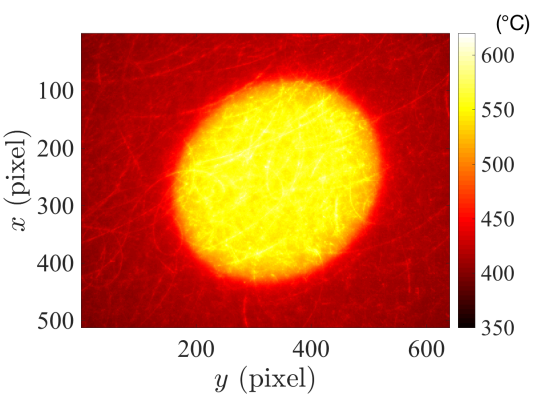

(b)

Figure 2: Typical example of reference (a) and deformed (b) IR images (color bar in degrees $\mathrm{C}$ ). The space coordinates $x$ and $y$ are expressed in pixels (1 pixel $=15 \mu \mathrm{m}$ )

The use of gray level residual error will be used in the sequel to provide an image based information on the quality of the DIC calculation. The instantaneous DIC residual $\rho(t)$ is calculated at time $t$ as

$$
\rho(t)=\frac{\left\langle\left(\widetilde{f}(\boldsymbol{x}, t)-f_{0}(\boldsymbol{x})^{2}\right\rangle_{R O I}^{1 / 2}\right.}{\left(\max _{R O I}\left(f_{0}\right)-\min _{R O I}\left(f_{0}\right)\right)}
$$

where $\langle\bullet\rangle$ denotes spatial averages. The global residual considers the time integral of $\rho(t)$

$$
\hat{\boldsymbol{\rho}}=\frac{\sum_{\boldsymbol{t} \in \tau} \rho(t)}{T}
$$

where $T$ denotes the number of considered frames. The initial residual level corresponding to the two frames shown in Figure 2 is $120 \%$ while the global residual $\hat{\boldsymbol{\rho}}$ equals $70 \%$. 


\subsection{Thermal shock assessment}

The laser shock is simulated with the FE software Cast $3 \mathrm{~m}^{\mathrm{TM}}$ (Figure 3). First a heat transfer model is set up to determine the 3D temperature distribution during thermal shock. An initial calibration of the boundary conditions is performed thanks to the experimental IR frames, which provide the temperature distribution (after a suited calibration [31]). Let us mention that for the following use, one may tolerate even rough approximations for the spatial thermal boundary conditions of the problem without much consequence. In contrast, the boundary conditions for the temporal evolution are strictly followed.

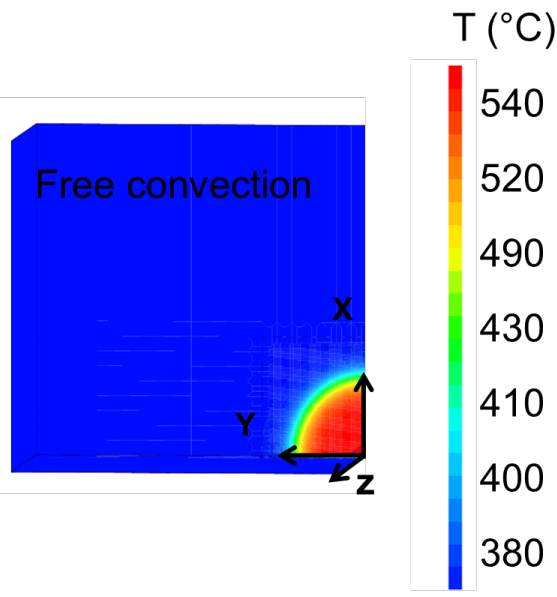

(a)

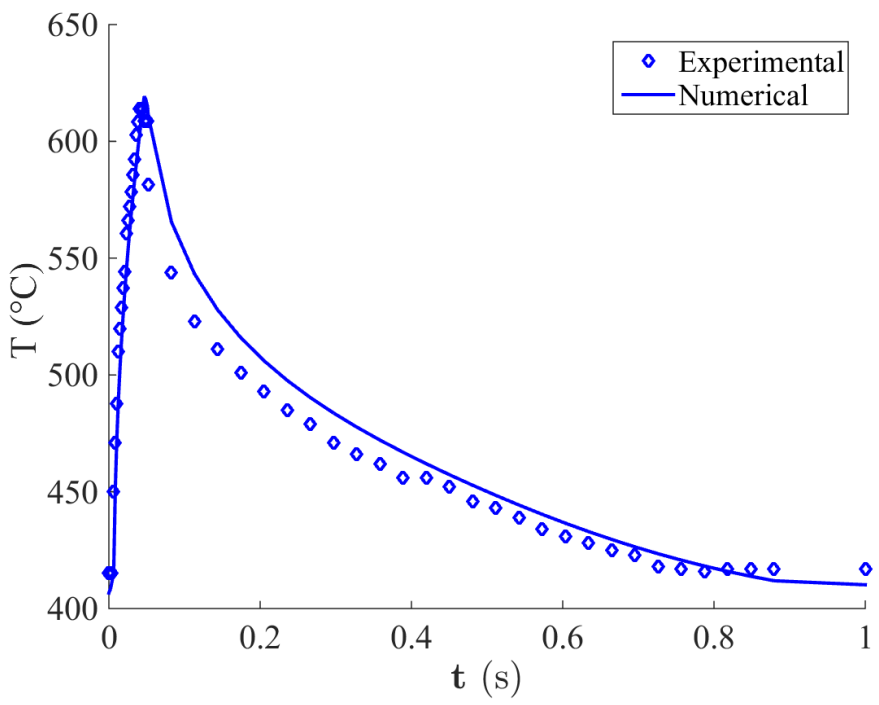

(b)

Figure 3: (a) Boundary conditions and (b) temporal histories provided by the numerical and experimental results at the center of the laser shock 
The mechanical response is modelled by prescribing the resulting $3 \mathrm{D}$ thermal field. A thermoelastoplastic constitutive law [39] with two kinematic hardenings is considered to describe the material behavior [37]. The predicted 2D surface displacement fields, projected onto the camera frame, are shown in Figure 4.

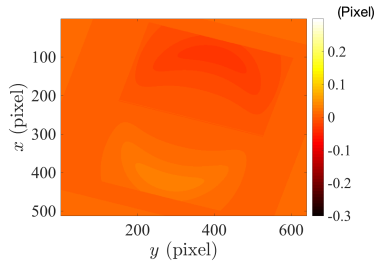

(a) $u_{x}$

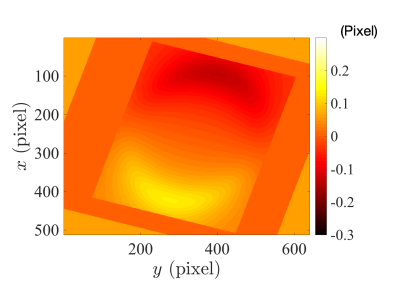

(d) $u_{x}$

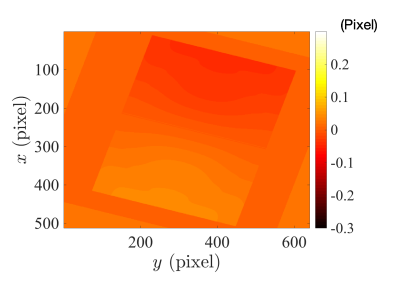

(g) $u_{x}$

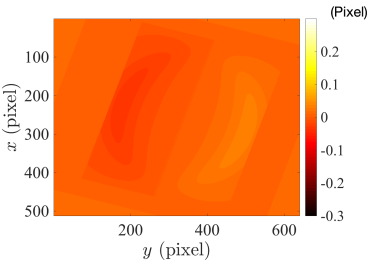

(b) $u_{y}$

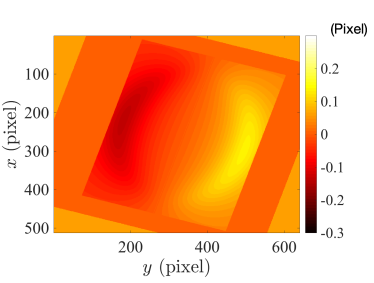

(e) $u_{y}$

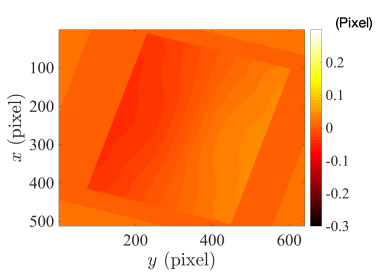

(h) $u_{y}$

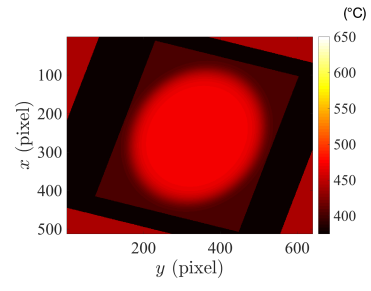

(c) $T$

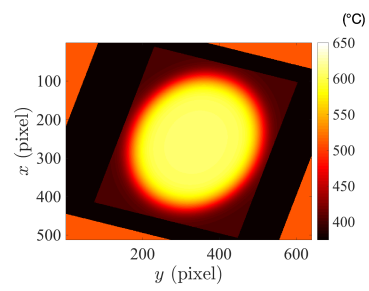

(f) $T$

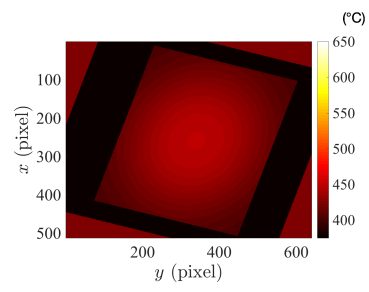

(i) $T$

Figure 4: $u_{x}(\mathrm{a}, \mathrm{d}, \mathrm{g})$ and $u_{y}(\mathrm{~b}, \mathrm{e}, \mathrm{h})$ components of the displacement fields (expressed in pixel) and temperature (c,f,i) fields at the beginning of the laser pulse $(a, b, c)$, and the end of laser pulse (d,e,f) and in the cooling part of the thermal cycle $(\mathrm{g}, \mathrm{h}, \mathrm{i})$. The numerical fields are projected onto the IR camera frame, $x$ and $y$ are expressed in pixels $(1$ pixel $=15 \mu \mathrm{m})$

\subsection{Extraction of temporal modes}

As above mentioned, KLD is performed independently for the temperature and the displacement fields. Because of symmetry, the spatiotemporal description of the displacement fields $u_{x}$ and $u_{y}$ provides the same temporal modes. Figure 5 shows the first three spatial modes for both fields (i.e., displacement and temperature). 


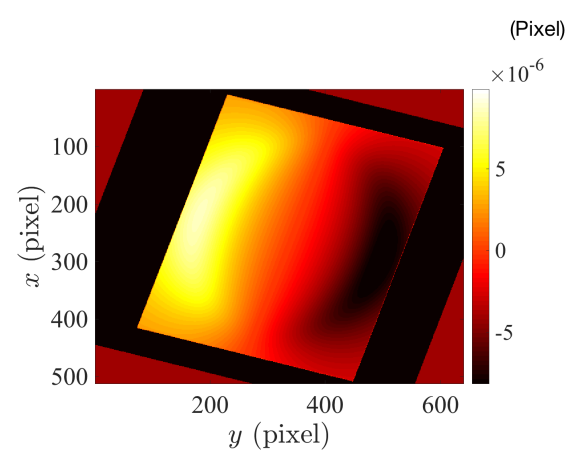

(a) $u_{y}$ mode 1

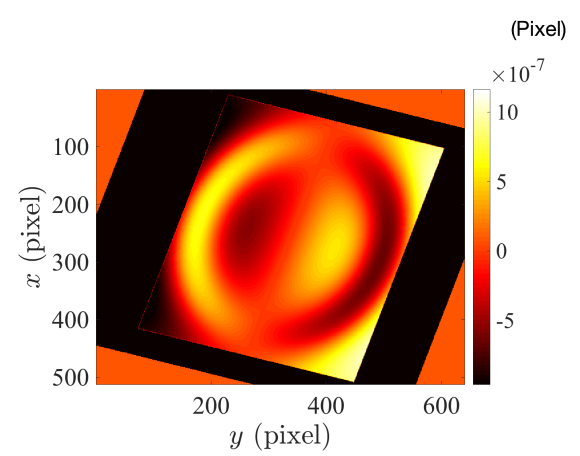

(c) $u_{y}$ mode 2

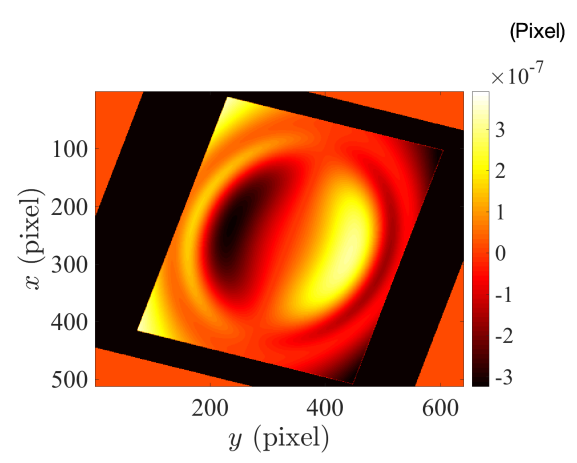

(e) $u_{y}$ mode 3

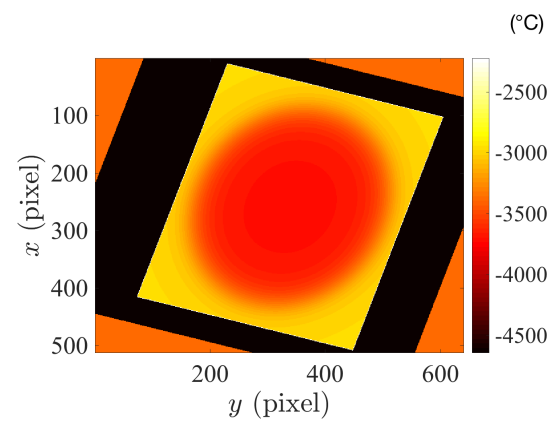

(b) $T$ mode 1

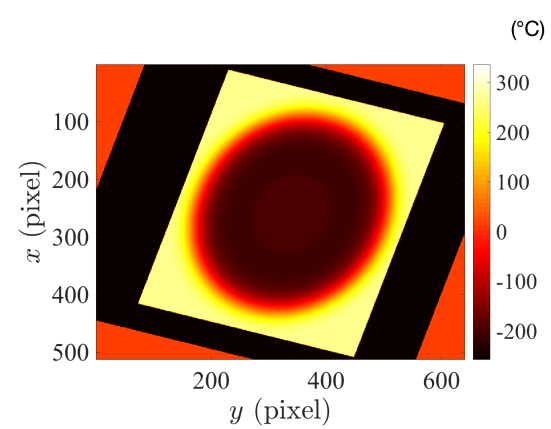

(d) $T$ mode 2

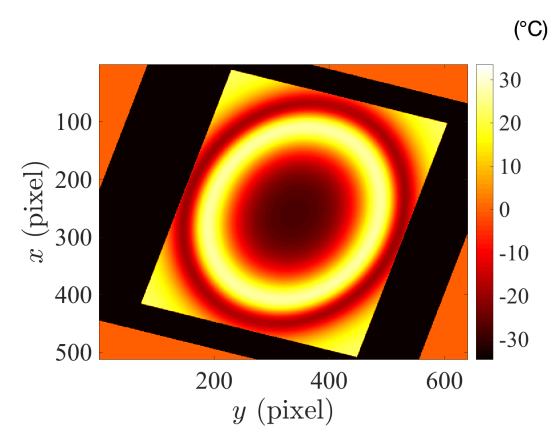

(f) $T$ mode 3

Figure 5: First three spatial modes obtained for the displacement fields component $u_{y}$ expressed in pixels (a,c,e), and temperature fields $T$ (b,d,f). The numerical fields are projected onto the IR camera space, $x$ and $y$ are expressed in pixels $(1$ pixel $=15 \mu \mathrm{m})$

The spatial modes could have been exploited to implement a (reduced model) I-DIC route for analyzing the experiment [37] where the GL corrections are performed by exploiting the spatial modes of the numerical temperature fields. However, small offsets are expected in the spatial modes (e.g., shape 
and profile of the laser spot may not be exact) and such model errors, although unimportant for the time history may preclude a proper spatial analysis. In the sequel, only the temporal modes extracted from the numerical predictions (and shown in Figure 6) are exploited.

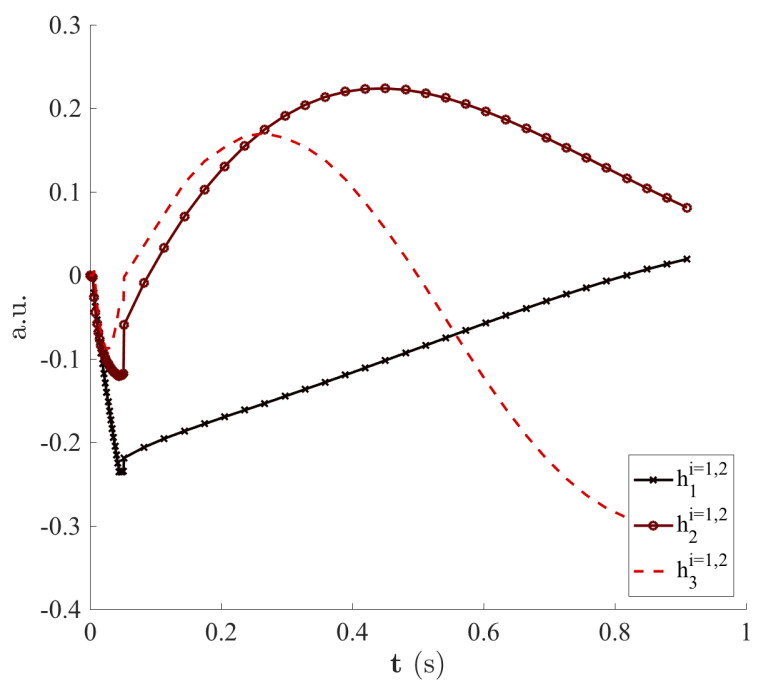

(a) Temporal modes for $u_{x}$

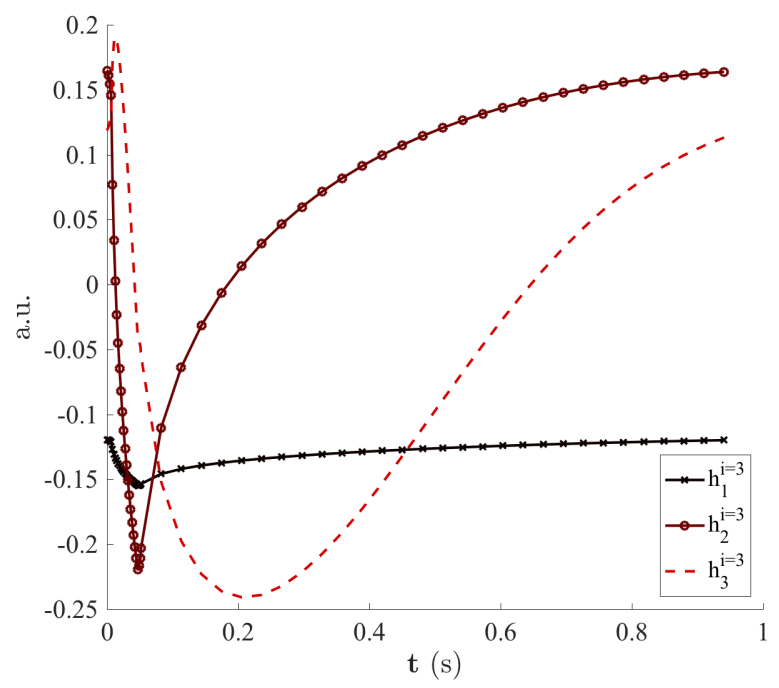

(b) Temporal modes for $T$

Figure 6: Three numerical temporal modes obtained for (a) $u_{x}$ components of the displacement field, and (b) temperature field

The choice of the number of modes is carried out by computing the normalized norm of the residuals obtained as the difference between the computed field and the approximation resulting from a truncation of the modal series. Figure 7 shows the change of these residuals against the truncation order at 
different instants. Less than four modes are sufficient to reconstruct the thermal fields at the beginning of the laser pulse ("t $=10 \mathrm{~ms}$ "), end of laser pulse (" $\mathrm{t}$ $=50 \mathrm{~ms}$ "), or in the cooling stage ("t $=80 \mathrm{~ms}$ "). The same trends are observed when a global residual ("Entire cycle") is considered.

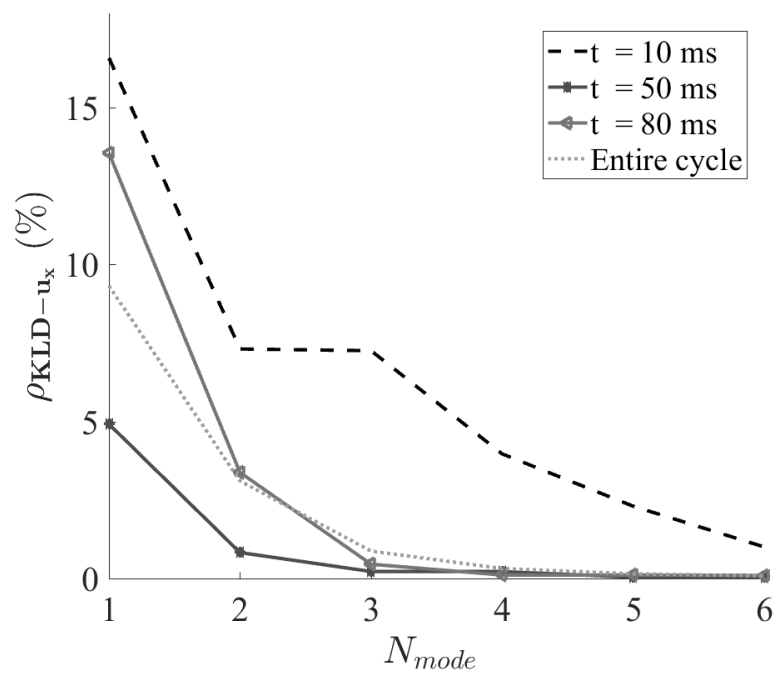

(a) Residuals for $u_{x}$

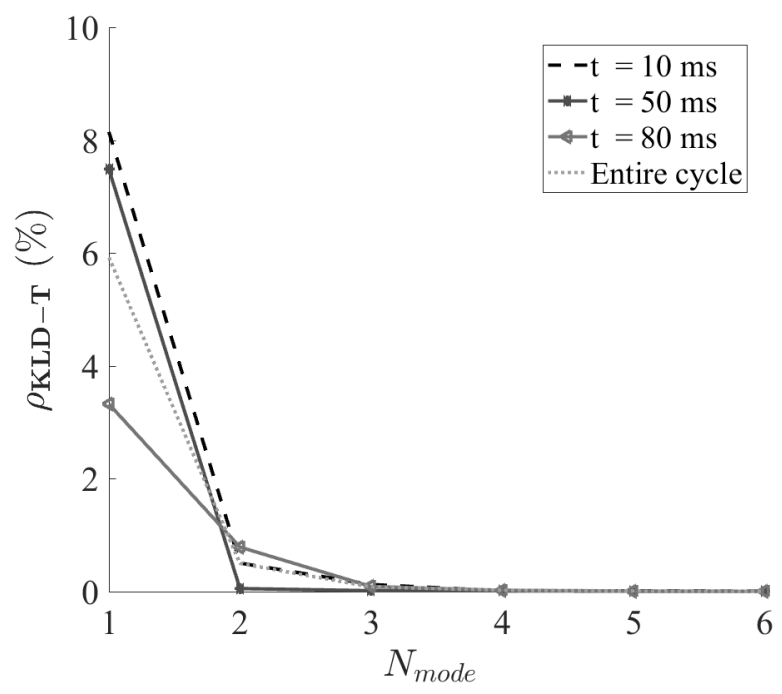

(b) Residuals for $T$

Figure 7: Residual, between the numerical and reconstructed results, change with the number of considered modes to reconstruct the numerical (a) $u_{x}$ component of the displacement and (b) $T$ temperature fields

The modal decomposition is performed on an uncoupled thermomechanical model, that is the mechanical response is assumed not to affect the thermal distribution. Such approximation is sufficient as the temperature elevation, which 
is caused by intrinsic dissipation due to plastic flow, is negligible compared with the heat generated by the laser pulse itself. Spatiotemporal DIC can still be used for coupled thermomechanical models. The numerical model would have to consider the coupling between heat transfer and mechanics. The final output of the FE model would still provide spatiotemporal temperature and displacement fields. They could be processed similarly via KLD.

\subsection{Modal DIC analysis}

The use of IR frames for DIC purposes [17,19] is made possible by considering in the formalism some additional (to displacement) corrections such as brightness and contrast, since their changes in space and time are equivalent to the effect of the temperature field. Therefore the temporal modes obtained from the computed temperature fields are exploited to determine such correction fields.

At convergence the measured DIC modes for gray level corrections and $u_{x}$ are displayed in Figure 8 and Figure 9 respectively. The GL modes are comparable to those determined numerically from the thermal model (see Figure 5). However for the displacement modes, only the first one is close to that of the simulation. The second and third modes are not trivially corresponding to those presented in Figure 5. However, the very low amplitude of variation may suggest that those modes are masked by the uncertainty, and thus only the first (experimental) mode really contains a trustworthy information. 
$\left({ }^{\circ} \mathrm{C}\right)$

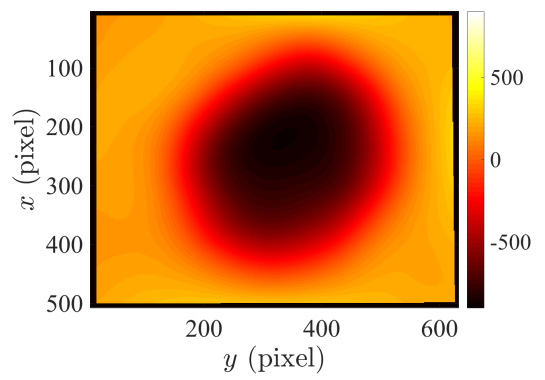

(a)

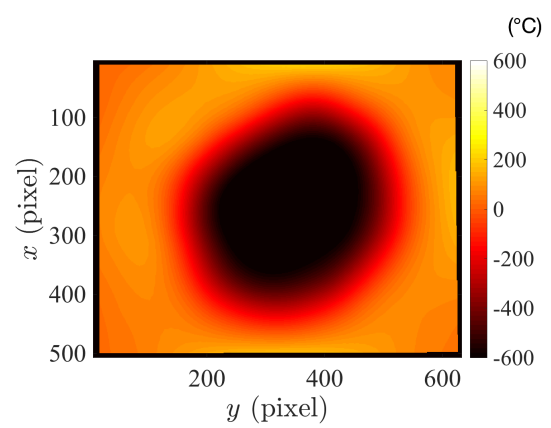

(c)

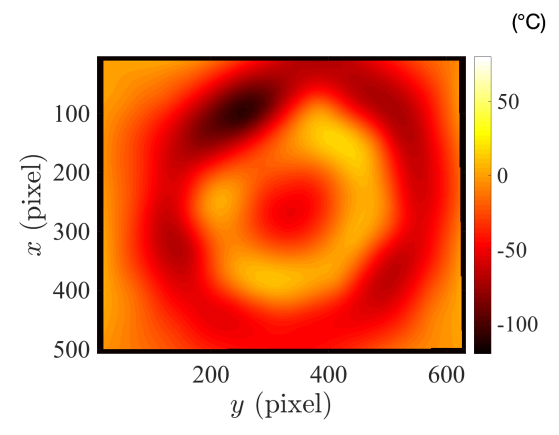

(e)

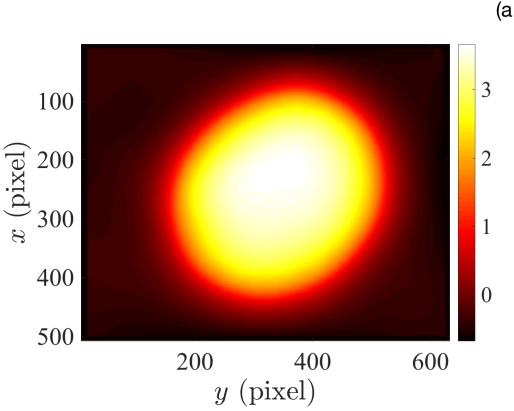

(b)

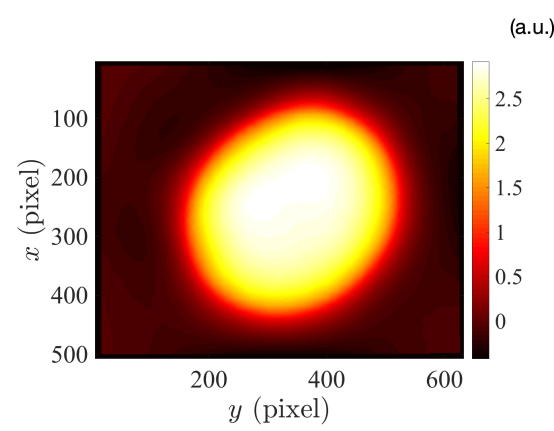

(d)

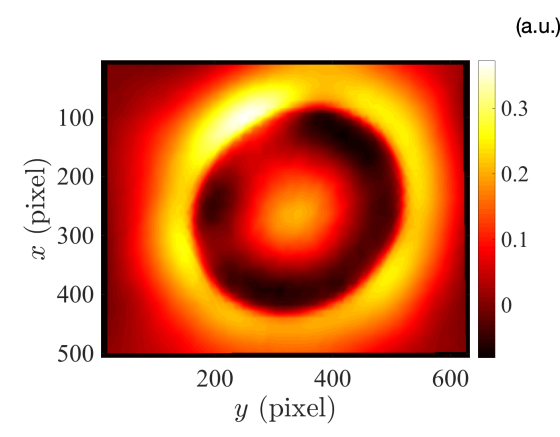

(f)

Figure 8: Three experimental spatial modes obtained for (a,c,e) brightness $a$ (expressed in ${ }^{\circ} \mathrm{C}^{1}$ ) and $(\mathrm{b}, \mathrm{d}, \mathrm{f})$ the contrast $b$ fields. The space coordinates $x$ and $y$ are expressed in pixels $(1$ pixel $=15 \mu \mathrm{m})$

\section{प}

${ }^{1}$ Here the brightness fields do not represent the temperature fields as they are combined with the contrast fields. The unit is still ${ }^{\circ} \mathrm{C}$ as the gray levels on the images were transformed, using the calibration file and emissivity value of the surface, in temperature fields. However, when brightness and contrast are used, the true temperature reads $T=a+(1+b) f_{0}$. 


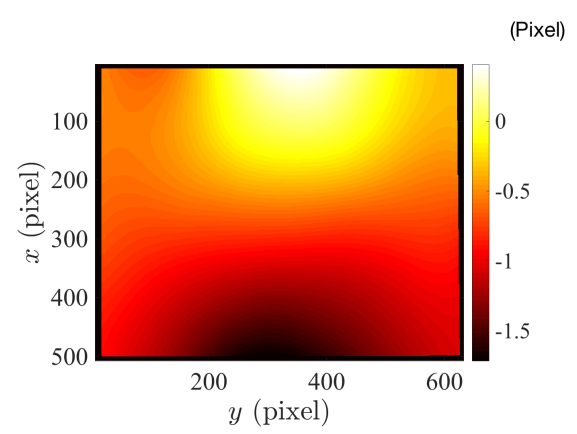

(a)

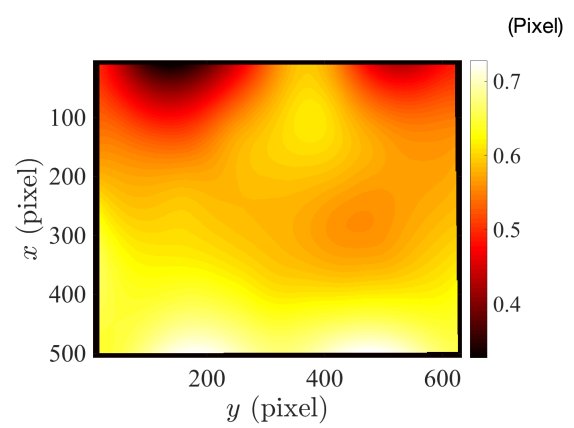

(c)

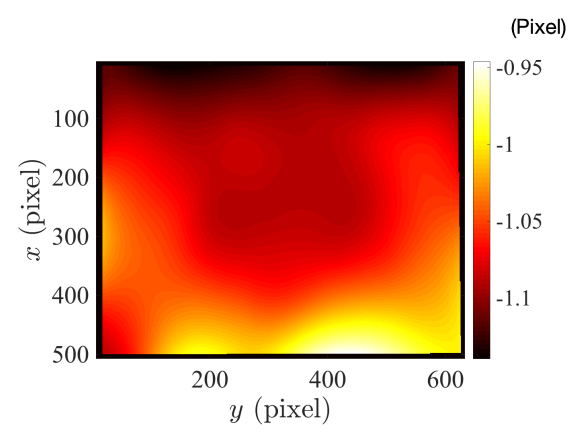

(e)

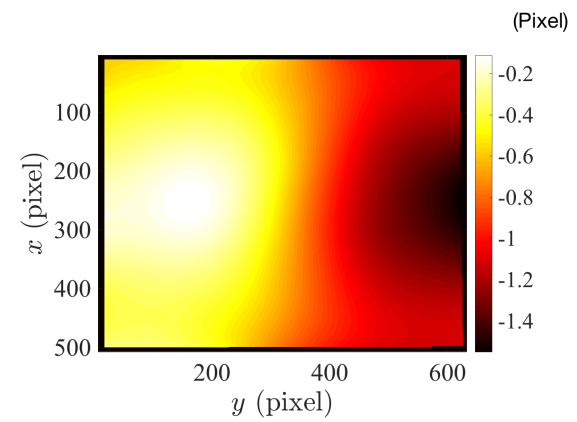

(b)

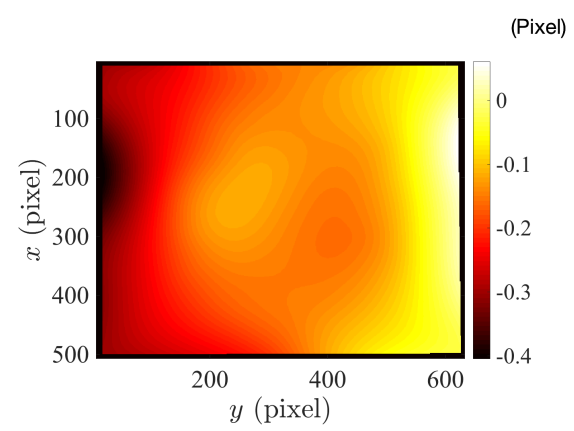

(d)

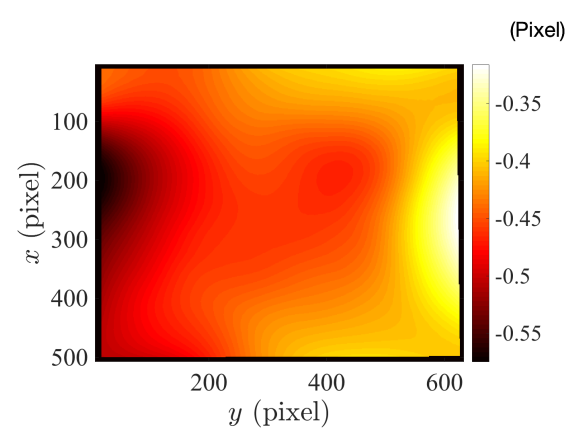

(f)

Figure 9: Three experimental spatial modes obtained for the displacement fields (a,c,e) $u_{x}$ and (b,d,f) $u_{y}$ (expressed in pixel). The space coordinates $x$ and $y$ are expressed in pixels $(1$ pixel $=15 \mu \mathrm{m})$

The combination of the determined spatial and temporal modes provides the full spatiotemporal description (see Figure 10). The biaxial state generated by the laser shock is recovered as expected [31], thereby corroborating the argument that the spatial content of modes 2 and 3 does not contribute much to the overall displacement field. This is the result of the competition between the mechanical reaction (compression) and thermal expansion that generate such laser shocks. 


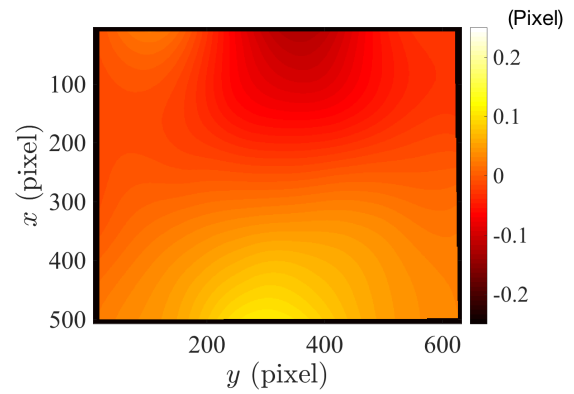

(a) $u_{x}$

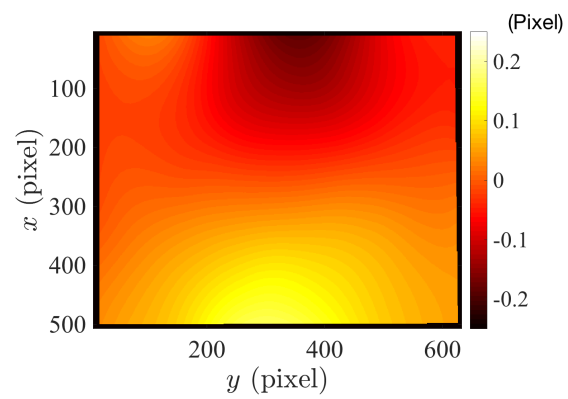

(c) $u_{x}$

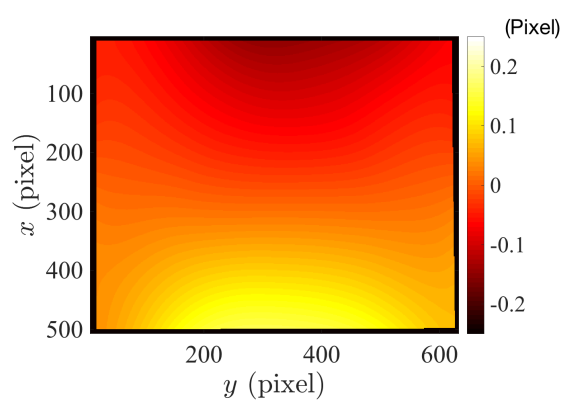

(e) $u_{x}$

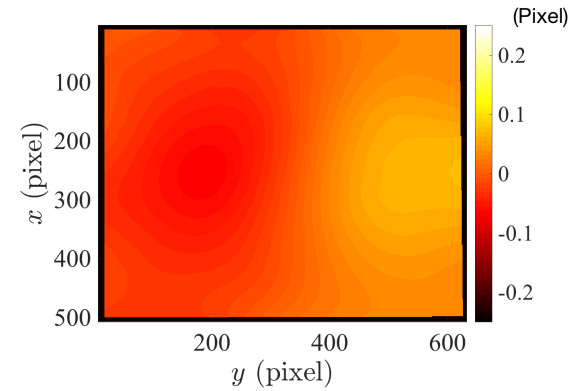

(b) $u_{y}$

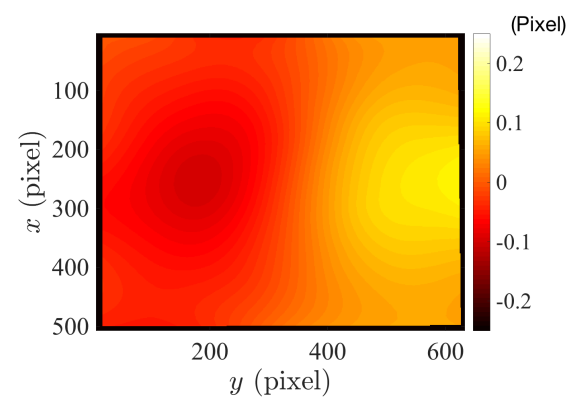

(d) $u_{y}$

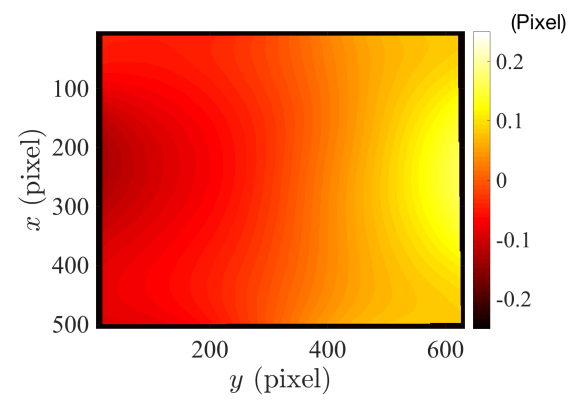

(f) $u_{y}$

Figure 10: Experimental $u_{x}$ and $u_{y}$ components of the displacement field (expressed in pixel) at the beginning of the laser pulse $(a, b)$, the end of the laser pulse $(\mathrm{c}, \mathrm{d})$ and in the cooling part of the thermal cycle $(\mathrm{e}, \mathrm{f})$. The space coordinates $x$ and $y$ are expressed in pixels $(1$ pixel $=15 \mu \mathrm{m})$

The corresponding brightness field correction $a$ and DIC residuals are shown in Figure 11. They describe well the temperature history. This is proven by the global DIC residual $\hat{\boldsymbol{\rho}}$ for which the level does not exceed $5 \%$. Calculation where only brightness is considered provide global DIC residual levels of $10 \%$. 


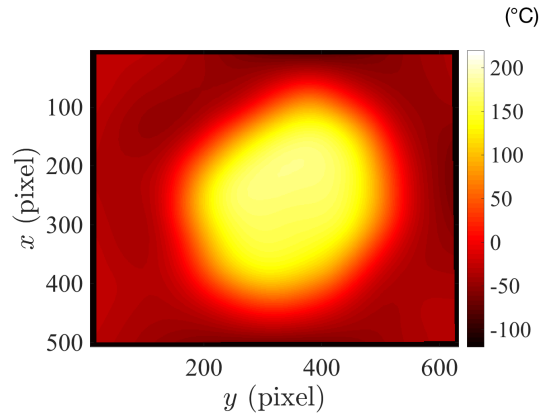

(a) Brightness $a$

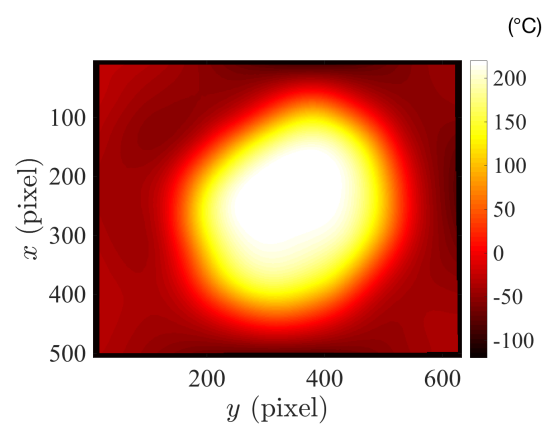

(c) Brightness $a$

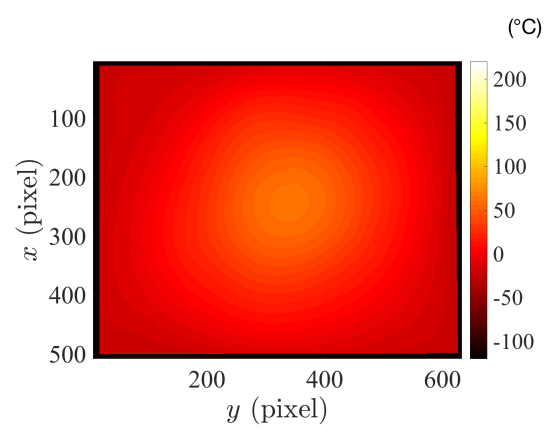

(e) Brightness $a$

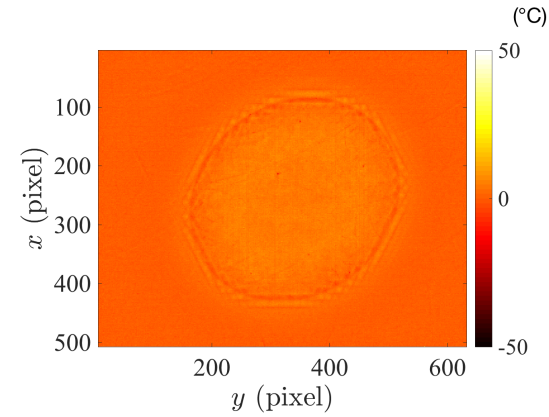

(b) Residual

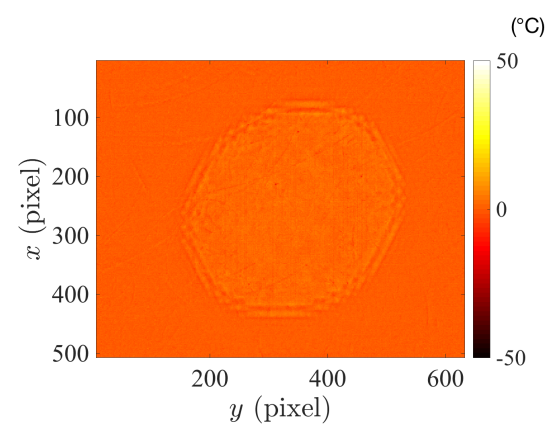

(d) Residual

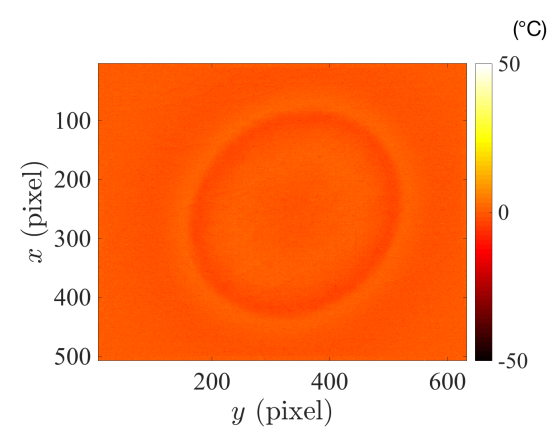

(f) Residual

Figure 11: Brightness correction $a$ and DIC residual fields at the beginning of the laser pulse $(\mathrm{a}, \mathrm{b})$, the end of the laser pulse $(\mathrm{c}, \mathrm{d})$ and in the cooling part of the thermal cycle $(\mathrm{e}, \mathrm{f})$. The space coordinates $x$ and $y$ are expressed in pixels $(1$ pixel $=15 \mu \mathrm{m})$

An analysis was also performed with no GL correction in the DIC approach. Only the spatiotemporal regularization for the kinematics was applied to the IR frames. The algorithm converged in few iterations (23) and the final residuals were about $60 \%$ (mainly due to the non conservation of brightness). However, in spite of such high levels of residuals, the overall shape of the displacement 
field was well captured. The amplitudes of displacement are much larger and not deemed trustworthy. An image-per-image approach diverges when no GL corrections are applied.

\subsection{Application to images perturbed by convection}

The thermal fatigue test is performed at high temperature (initial plate temperature $400^{\circ} \mathrm{C}$ ). The helium atmosphere in the chamber circulates all along the test to cool the walls. This flow generates a forced convection above the studied sample surface. The mixing of gas at different temperatures has the effect of generating "ghost" motions due to optical index variations. The effect of convection on the displacement fields is illustrated in Figure 12 when an image-per-image registration is performed with the visible light camera images. 


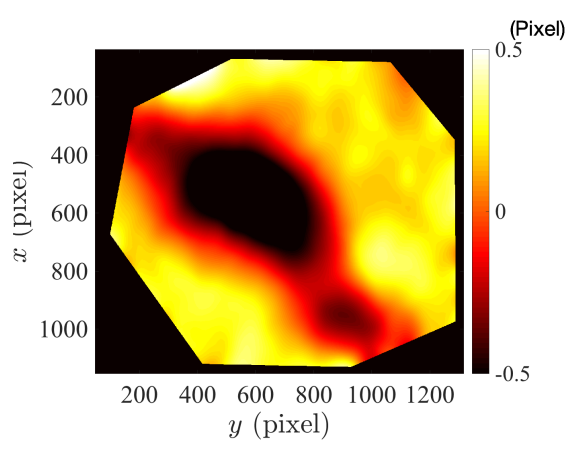

(a) $u_{x}$

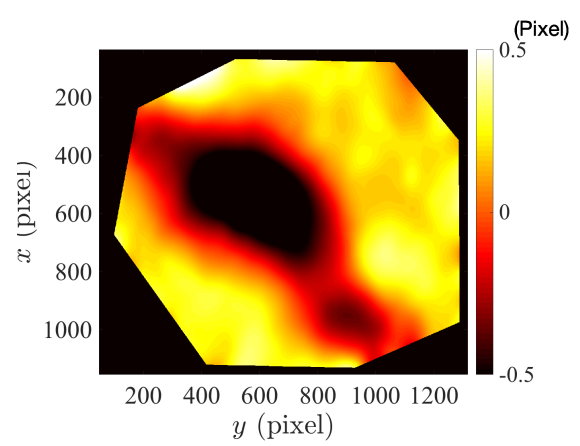

(c) $u_{x}$

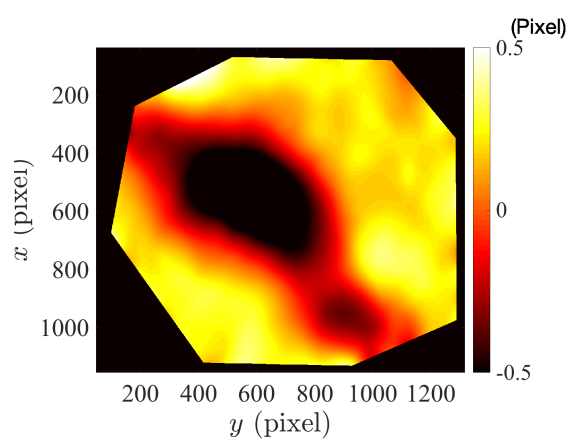

(e) $u_{x}$

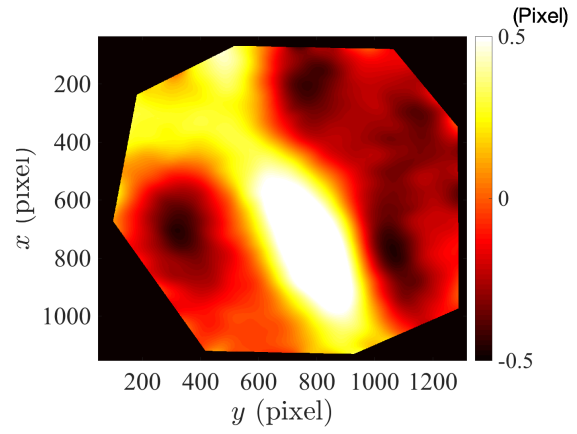

(b) $u_{y}$

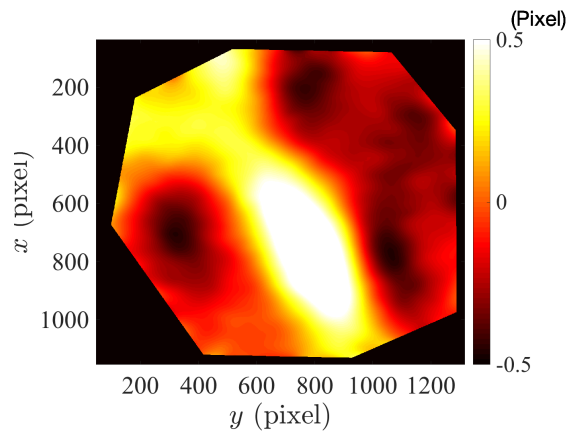

(d) $u_{y}$

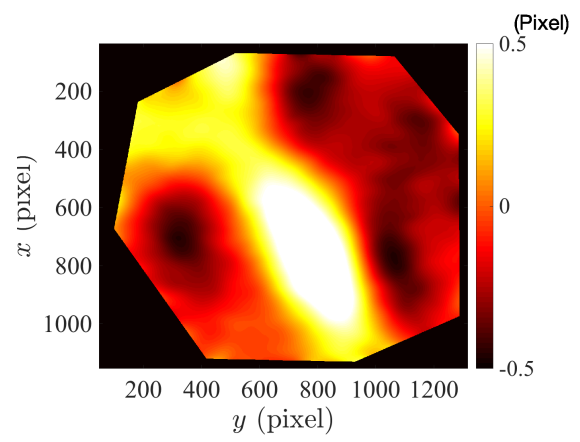

(f) $u_{y}$

Figure 12: Experimental $u_{x}$ and $u_{y}$ components of the displacement fields (expressed in pixel) at the beginning of the laser pulse $(\mathrm{a}, \mathrm{b})$, the end of the laser pulse $(\mathrm{c}, \mathrm{d})$, and in the cooling part of the thermal cycle $(\mathrm{e}, \mathrm{f})$. These fields are obtained with an image-per-image DIC approach while convection is occurring during image acquisition. The space coordinates $x$ and $y$ are expressed in pixels $(1$ pixel $=10 \mu \mathrm{m})$

The displacement fields are very different from those expected from the FE simulation (Figure 4), and from the previous experimental analysis (Figure 10). The displacement fields due to the thermomechanical response and optical dis- 
tortion are correlated rendering the interpretation difficult. The brightness and residual fields are shown in Figure 13. The brightness correction is not necessary for visible light camera images but can still be performed as the CMOS sensor is sensitive to the presence of the heat source generated by the laser. The DIC residuals maps appear as pure noise and show that DIC performed well, although one cannot distinguish between the physical displacement field and the spurious motions due to convection. 


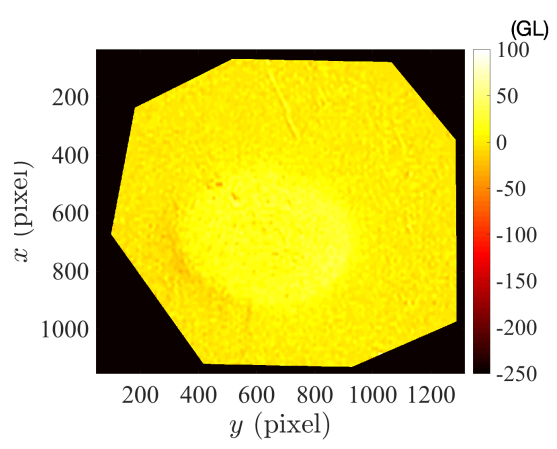

(a) Brightness $a$

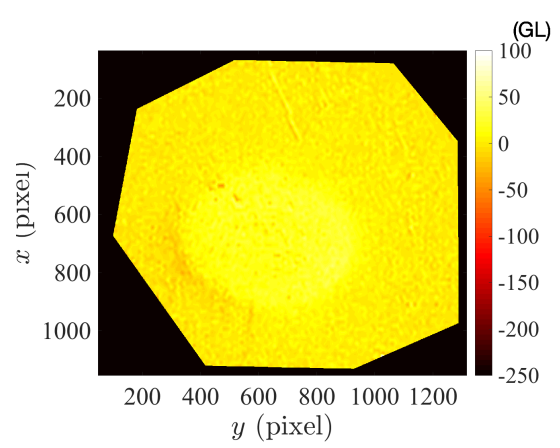

(c) Brightness $a$

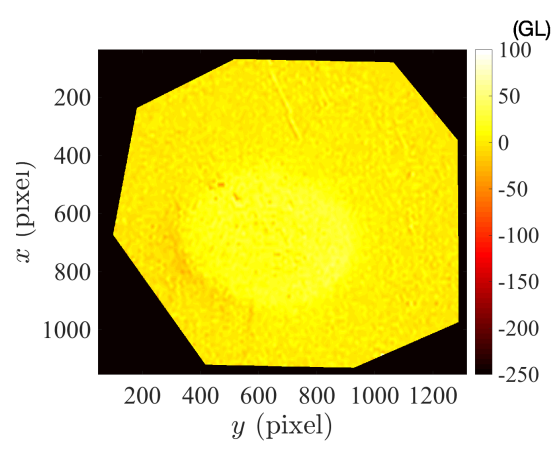

(e) Brightness $a$

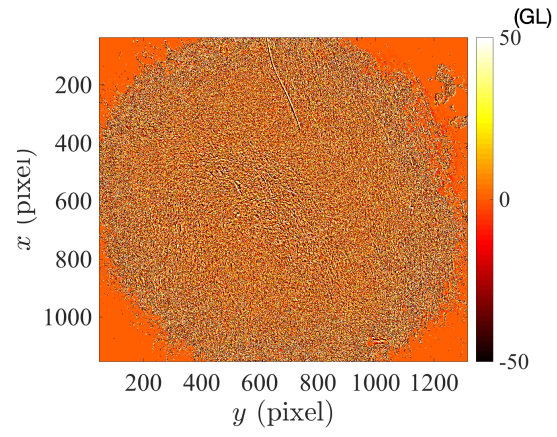

(b) Residual

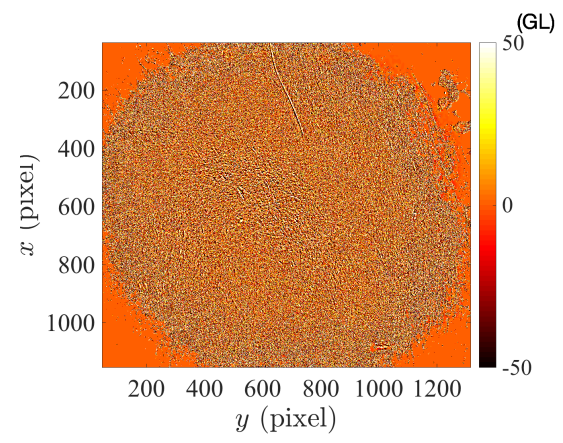

(d) Residual

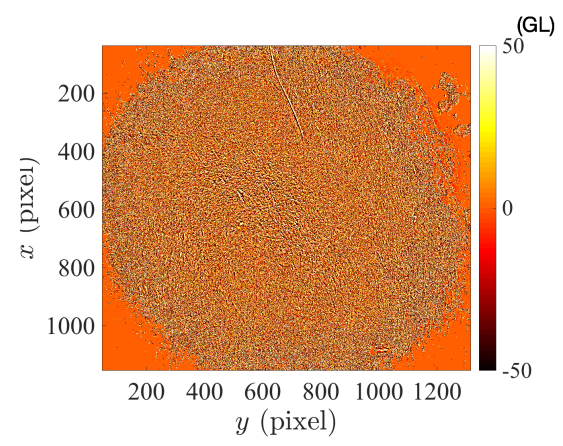

(f) Residual

Figure 13: The brightness correction $a$ and DIC residual fields (expressed in gray level (GL)) at the beginning of the laser pulse $(\mathrm{a}, \mathrm{b})$, the end of the laser pulse $(\mathrm{c}, \mathrm{d})$, and in the cooling part of the thermal cycle $(\mathrm{e}, \mathrm{f})$. These fields are obtained with an image-per-image DIC approach while convection is occurring during image acquisition. The space coordinates are $x$ and $y$ are expressed in pixels $(1$ pixel $=10 \mu \mathrm{m})$

One pragmatic way to circumvent such effect is simply to shut down helium flow and the convection has much less impact on the images. However, in cases where the thermal shock frequency exceeds $1 \mathrm{~Hz}$, the helium gun has to be on 
during the entire test including image acquisition. This seems to preclude the use of DIC in those cases. However, the displacement induced by convection is not expected to follow the temporal modes extracted from the thermal model. Hence, by considering only the displacement field that is consistent with the model-based temporal modes, it is expected that the effect of convection will be drastically reduced, thereby opening new perspectives for exploiting high frequency laser shock tests.

A paradox is then observed, namely, the global DIC residual obtained with the spatiotemporal regularization is higher than that obtained without any temporal penalization while in the latter case the displacement fields do not correspond to the expected ones. The residual levels of $\rho(t)$ are in the range $1-3 \%$ while they do not exceed $0.5 \%$ when no temporal regularization is applied. This is mainly due to the expression of the fictitious displacement modes generated by the convection on the images. As they are not captured by the spatiotemporal approach, because they do not represent any thermomechanical signature, they are transcribed into the residuals fields.

The measured displacement fields after spatiotemporal regularization are shown in Figure 14. The biaxial state is recovered and the convection effect is cleared for the most part. The temporal modes successfully extract the consistent contribution of the displacement field. 


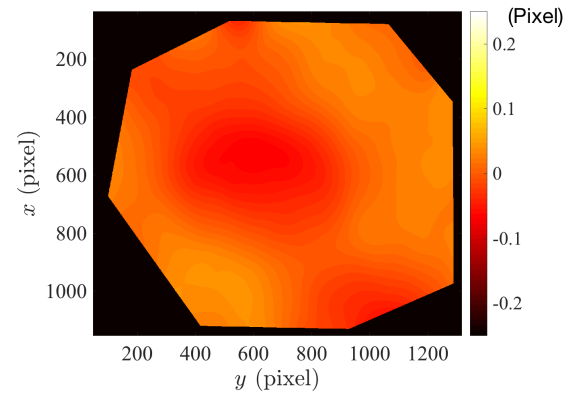

(a) $u_{x}$

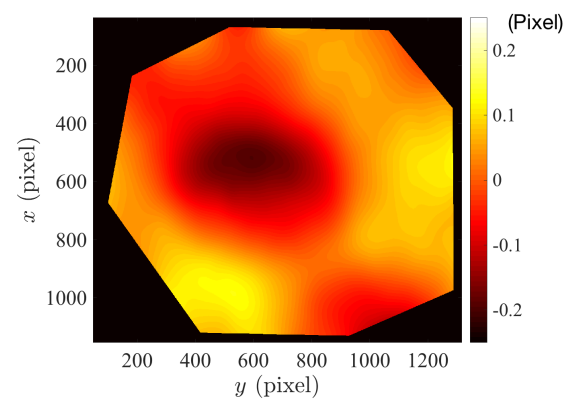

(c) $u_{x}$

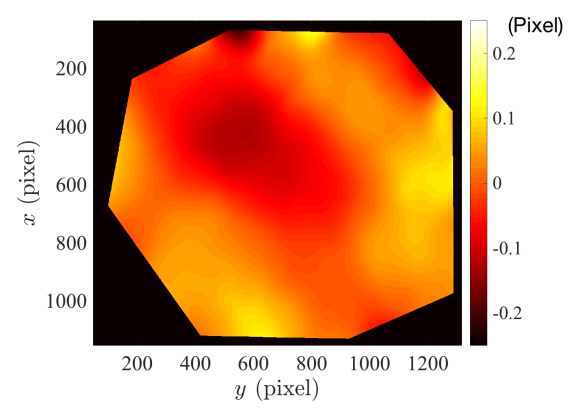

(e) $u_{x}$

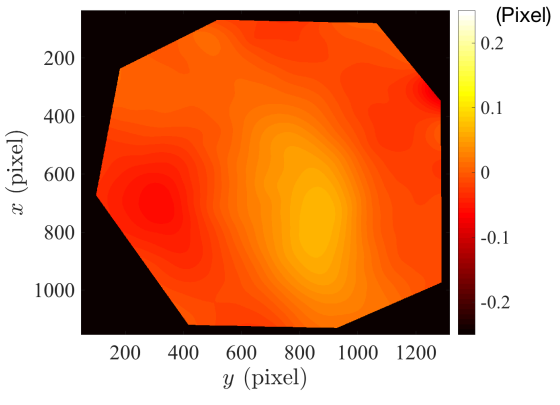

(b) $u_{y}$

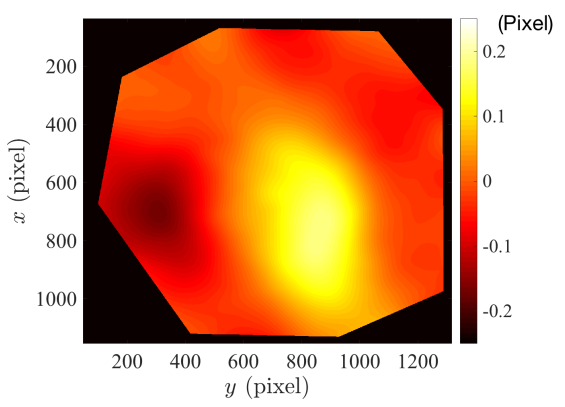

(d) $u_{y}$

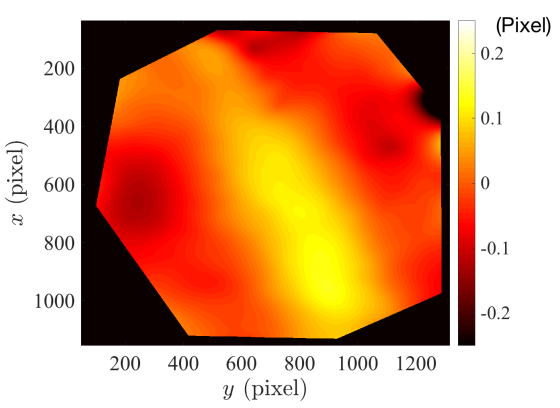

(f) $u_{y}$

Figure 14: Experimental $u_{x}$ and $u_{y}$ components of the displacement fields (expressed in pixel) at the beginning of the laser pulse $(\mathrm{a}, \mathrm{b})$, the end of the laser pulse $(\mathrm{c}, \mathrm{d})$, and in the cooling part of the thermal cycle $(\mathrm{e}, \mathrm{f})$. These fields are obtained with the space/time DIC approach while convection is occurring during image acquisition. The space coordinates $x$ and $y$ are expressed in pixels (1 pixel $=10 \mu \mathrm{m})$

It is important for the success of this strategy that the temporal mode of the convection part of the displacement field has a small projection (ideally null) with respect to those of the physical displacement. This approach somehow 
extends the strategy used for fighting the adverse effect of turbulent thermal plumes in high temperature mechanical tests that consisted in averaging images with a long exposure time [40]. In the latter case the time average of convective displacements is expected to be null, whereas in the present study, it is expected to be orthogonal to the model-based dominant temporal evolution. The effects of convection on images are still present but not captured by the constrained kinematics. This is illustrated on the residual maps shown in Figure 15. 


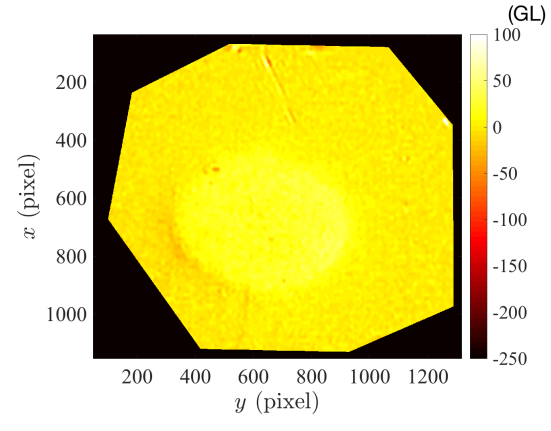

(a) $u_{x}$

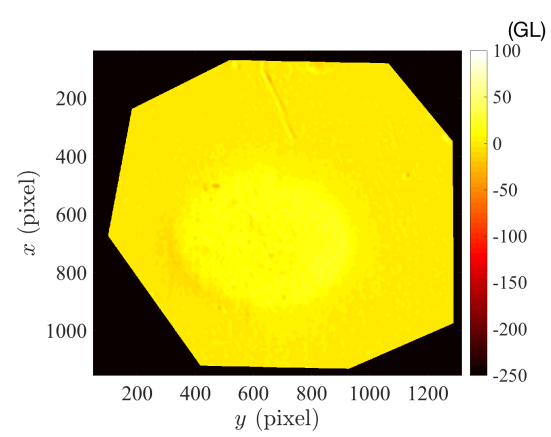

(c) $u_{x}$

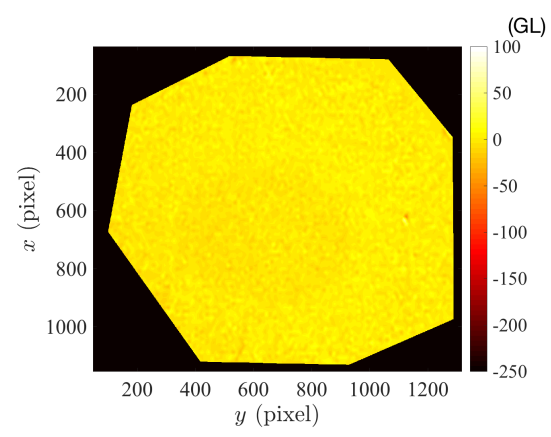

(e) $u_{x}$

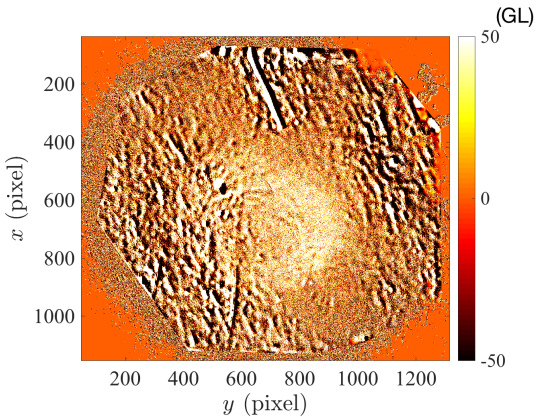

(b) $u_{y}$

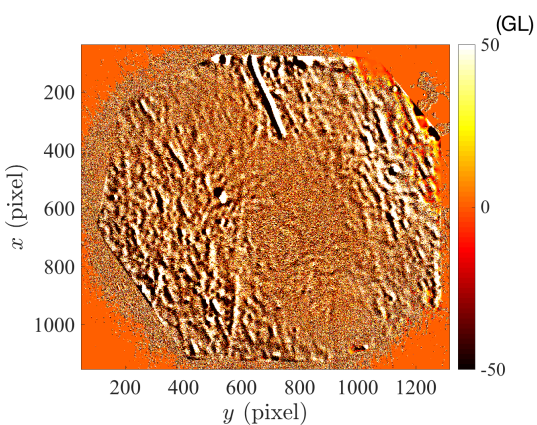

(d) $u_{y}$

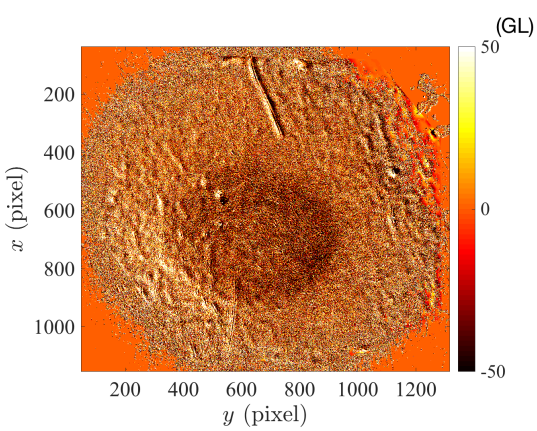

(f) $u_{y}$

Figure 15: Brightness correction $a$ and DIC residual fields (expressed in gray level (GL) at the beginning of the laser pulse $(a, b)$, the end of the laser pulse $(\mathrm{c}, \mathrm{d})$, and in the cooling part of the thermal cycle $(\mathrm{e}, \mathrm{f})$. These fields are obtained with the space/time DIC approach while convection is occurring during image acquisition. The space coordinates $x$ and $y$ are expressed in pixels $(1$ pixel $=$ $10 \mu \mathrm{m})$ 


\section{Frame pruning}

One of the benefits of the present DIC approach is an enhanced redundancy as the number of degrees of freedom is reduced to be able to capture the spatiotemporal displacement field with only a few modes (here 3 ). This is advantageous both for memory usage and time calculation saving. This advantage can actually be further optimized at the experimental level. It is proposed herein to perform "data pruning" consisting in ignoring entirely some frames in the analysis. In the present subsection, only a reduced number of frames $N_{\text {frame }}$ is kept in the otherwise untouched space/time DIC approach. Several downsamplings in time of IR frames are performed to compute the full displacement and temperature fields whereby $N_{\text {frame }}=25,12,6$ and 3 as compared to the entire sequence composed of 50 frames. The selected frames in the thermal shock history are shown in Figure 16. In the case when only three frames are exploited, two different choices of image numbers are analyzed. This will illustrate the importance of the instant selection for such limit case. The results will be compared to the case where the full movie is used in the same DIC approach.

Let us stress here that even if some frames are not exploited, using to modes allows the temperature and displacements to be determined at all pixels and time steps. The modes themselves provide an interpolation that is based on the physical model. This is much more trustworthy than the recourse to a priori defined shape functions in space or time. Moreover, as these modes are tailored to the studied case, the use of only a few degrees of freedom allows for the present discussion of pruning. When much more degrees of freedom are introduced, frame pruning becomes soon unstable (i.e., ill-conditioned).

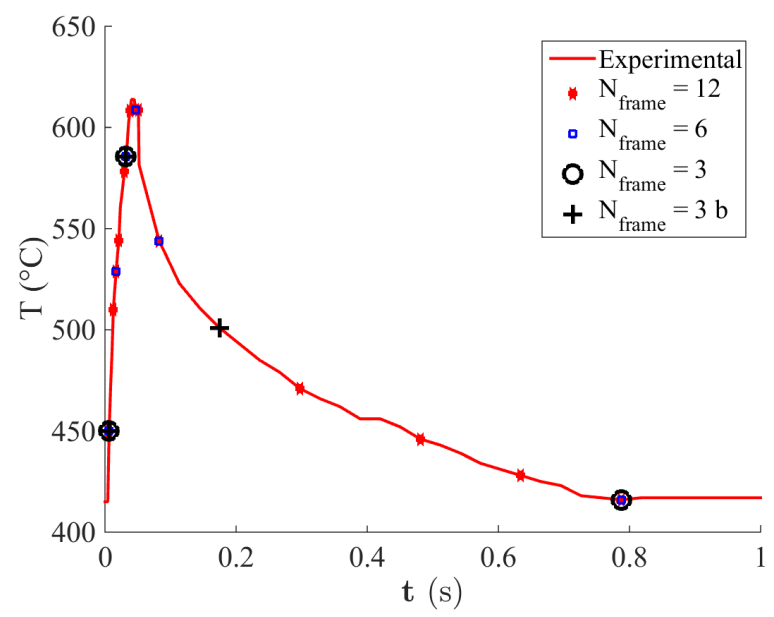

Figure 16: Frames selected for different optimization strategies

The spatial modes obtained for different $N_{\text {frame }}$ are all very similar to those shown in Figure 3. Some differences are observed mostly in terms of amplitudes and impact the DIC residual levels. The temperature variations and the DIC residual levels are presented in Figure 17. For the first case where $N_{\text {frame }}=3$ 
the thermal loading history is not well captured. This is due to the fact that two of the selected frames are at about the same temperature (see Figure 16), and hence the identification of the mode becomes ill-conditioned. When the three images are selected at different temperatures as performed for the case $N_{\text {frame }}=3 b$ the temperature variation is much better estimated and residuals are dramatically improved, although no more than 3 instants are used.

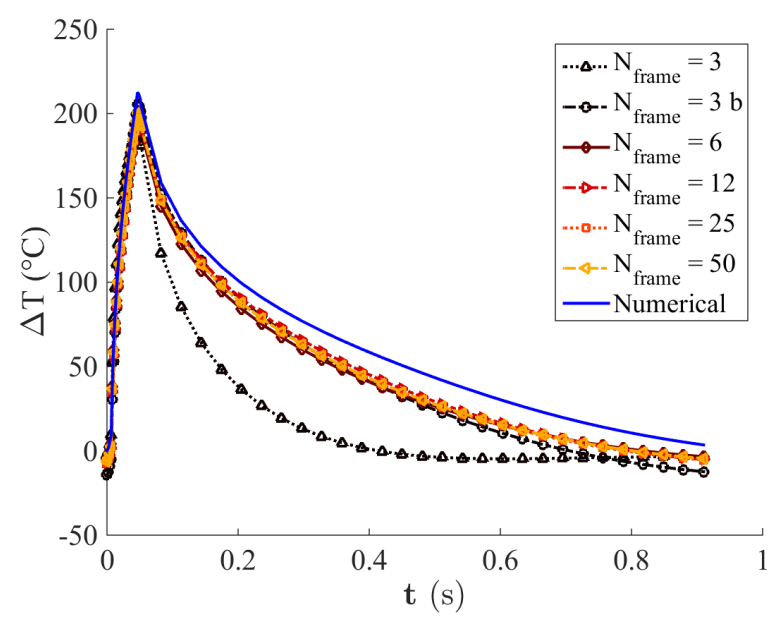

Figure 17: Time history of the measured temperature variations for different numbers $N_{\text {frame }}$ of used IR frames in the DIC calculation

For case 3b, and for a larger number of selected frames, the residuals drop down to levels comparable to the entire sequence (ca. 5-10\%). Only a very modest benefit is observed as $N_{\text {frame }}$ is increased, and the temperature time profile appears to be perfectly captured from $N_{\text {frame }}=6$ and more. The residual levels are mostly controlled by the GL corrections and hence it is difficult to read properly the effect of pruning frames on displacement measurements. Thus it is proposed to compare the displacement fields with the results obtained when the full movie is exploited, as shown in Figure 18. 


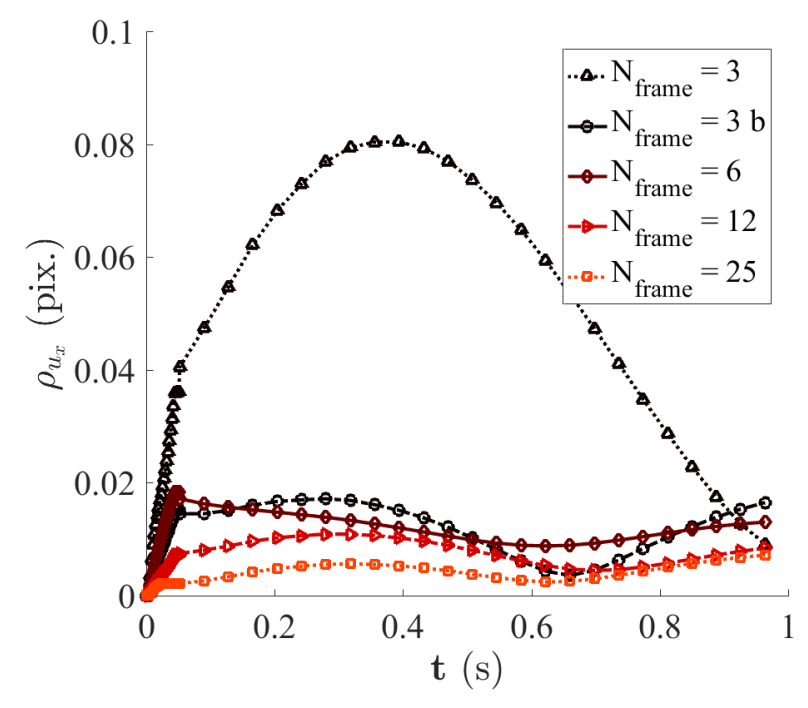

Figure 18: RMS differences between the measured displacement fields using $N_{\text {frame }}$ and that measured with the entire sequence. Displacements are expressed in pixels $(1$ pixel $=15 \mu \mathrm{m})$

The RMS displacement difference from the result obtained with a subset of frames as compared with the full sequence analysis shown in Figure 18. When $N_{\text {frame }}=3$, the difference can reach $8 \cdot 10^{-2}$ pixel. However, when a better selection is performed, $N_{\text {frame }}=3 b$, the residual levels decrease and the largest gaps are about $2 \cdot 10^{-2}$ pixel. When $N_{\text {frame }}=6$ and above, similar results are obtained, with a discrepancy less than 2 centipixels.

The number of selected frames has also a drastic impact on the required number of iterations to convergence (Table 1). It is observed that convergence is very slow when only three frames are selected (i.e., more than five times that of the entire series). This may be due to a degenerate mode not being identifiable. As more frames are included, the number of iterations drops down from about three times to one as $N_{\text {frame }}$ increases from 6 to 25 . It is noteworthy that from 25 to 50 frames, the number of iterations is close to identical and hence computation time is reduced by a factor of two, with virtually no prejudice to the registration quality.

Table 1: Number of iterations, $N_{i t e r}$, at convergence as a function of the number of chosen frames, $N_{\text {frame }}$

\begin{tabular}{|c|c|}
\hline$N_{\text {frame }}$ & $N_{\text {iter }}$ \\
\hline 3 & 135 \\
$3 \mathrm{~b}$ & 119 \\
6 & 68 \\
12 & 57 \\
25 & 22 \\
50 & 23 \\
\hline
\end{tabular}


From all these observations, two exploitations can be considered:

- Either the entire time series has been acquired and one wants to use it to exhaust all the information it contains. In that case, one may consider frame pruning only as an intermediate computation stage. For instance, cutting one image out of two speeds up the computation by a factor of two and reaches virtually the same solution. Once it has been achieved, it may be used as an initialization considering the entire series, so that about one or two iterations only are expected to be needed. This route drastically speeds up the entire computation. This principle can be used recursively, as small $N_{\text {frame }}$ are computationally cheap.

- Or one is limited by the total number of frames to be captured (e.g., for long-term fatigue tracking). In that case, it is important to save on the total number of frames to be acquired so that the saved images can be reinvested to get intermediate evolution stages.

In both cases, even if redundancy is partly sacrificed through frame pruning, the number of unknowns is still much smaller than the number of observables and hence quality factors (e.g., from residuals) can always be computed to assess the validity (or invalidity) of the procedure. Let us finally note that for extreme frame number reduction, one may hit difficulties in evaluating amplitudes (as observed for case $N_{\text {frame }}=3$ versus $N_{\text {frame }}=3 b$ ). The question of the optimal selection of the frames to be retained is an interesting question that warrants further consideration.

\section{Conclusions and prospects}

A global space/time DIC approach has been applied to the investigation of pulsed laser shock cyclic loading. Model-reduction techniques (i.e., KLD) are used to capture relevant temporal modes from physics-based thermal and mechanical models. The modal representation has been simply imported within the DIC framework to achieve a robust and accurate analysis. Challenging experimental cases were selected to test the robustness of the proposed methodology.

In the first case, very large gray level corrections occurred due to temperature variations. The spatiotemporal approach is very well suited to these corrections, and using KLD, no more than three modes revealed sufficient to account for the full space-time response for both temperature and displacement fields. In spite of the reduced number of degrees of freedom, residuals were brought down to 5-10\% (such values are to be compared to raw differences that can reach $120 \%$ as compared to the reference state).

In the second test case, convection induces spurious displacement fields due to optical index heterogeneities. It was observed that when constrained to follow the time evolution of the model, the DIC approach was able to partly retrieve the meaningful contributions from the apparent displacement field.

Last, it was shown that the constraints due to time regularization lead to the possibility to perform frame "pruning," with very limited impact on the result quality for $N_{\text {frame }}$ as low as 3 or 6 as compared to 50 frames in the full 
sequence. This saving may allow for a better coverage of the fatigue test over long times, or it can be used to speed up convergence time of DIC, temporarily within the algorithm, without further consequence on the final result.

\section{References}

[1] M. A. Sutton, W. J. Wolters, W. H. Peters, W. F. Ranson, and S. R. McNeill, "Determination of displacements using an improved digital correlation method," Image and Vision Computing, vol. 1, pp. 133-139, Aug. 1983.

[2] T. C. Chu, W. F. Ranson, and M. A. Sutton, "Applications of digital-imagecorrelation techniques to experimental mechanics," Experimental Mechanics, vol. 25, no. 3, pp. 232-244, 1985.

[3] S. McNeill, W. Peters, and M. Sutton, "Estimation of stress intensity factor by digital image correlation," Engineering Fracture Mechanics, vol. 28, pp. 101-112, Jan. 1987.

[4] H. A. Bruck, S. R. McNeill, M. A. Sutton, and W. H. Peters, "Digital image correlation using Newton-Raphson method of partial differential correction," Experimental Mechanics, vol. 29, no. 3, pp. 261-267, 1989.

[5] M. Grédiac, and F. Hild, eds., Full-Field Measurements and Identification in Solid Mechanics. London (UK): ISTE / Wiley, 2012.

[6] B. D. Lucas, and T. Kanade, "An Iterative Image Registration Technique with an Application to Stereo Vision," pp. 674-679, 1981.

[7] F. Hild, and S. Roux, "Digital Image Correlation," in Optical Methods for Solid Mechanics : A Full-Field Approach, Wiley-VCH, Berlin (Germany). P. Rastogi and Editor E. Hack (Edts.), 2012, pp. 183-228.

[8] M. A. Sutton, J. Orteu, and H. W. Schreier, Image Correlation for Shape, Motion and Deformation Measurements. Springer, 2009.

[9] W. Tong, "Formulation of Lucas-Kanade Digital Image Correlation Algorithms for Non-contact Deformation Measurements: A Review," Strain, vol. 49, no. 4, pp. 313-334, 2013.

[10] G. Besnard, F. Hild, and S. Roux, "'Finite-Element" Displacement Fields Analysis from Digital Images: Application to Portevin-Le Châtelier Bands," Experimental Mechanics, vol. 46, no. 6, pp. 789-803, 2006.

[11] S. Ma, Z. Zhao, and X. Wang, "Mesh-based digital image correlation method using higher order isoparametric elements," J. Strain Analysis, vol. 47, no. 3, pp. 163-175, 2012.

[12] P. Cheng, M. Sutton, H. Schreier, and S. McNeill, "Full-field speckle pattern image correlation with b-spline deformation function," Exp. Mech., vol. 42, no. 3, pp. 344-352, 2002. 
[13] J. Réthoré, T. Elguedj, P. Simon, and M. Coret, "On the Use of NURBS Functions for Displacement Derivatives Measurement by Digital Image Correlation," Experimental Mechanics, vol. 50, no. 7, pp. 1099-1116, 2010.

[14] A. N. Tikhonov, and V. Y. Arsenin, Solutions of Ill-posed problems. New York: J. Wiley, 1977.

[15] J. Réthoré, S. Roux, and F. Hild, "An extended and integrated digital image correlation technique applied to the analysis of fractured samples," European Journal of Computational Mechanics, vol. 18, pp. 285-306, Jan. 2009.

[16] Z. Tomičević, F. Hild, and S. Roux, "Mechanics-aided digital image correlation," The Journal of Strain Analysis for Engineering Design, vol. 48, no. 5, pp. 330-343, 2013.

[17] A. Maynadier, M. Poncelet, K. Lavernhe-Taillard, and S. Roux, "One-shot Measurement of Thermal and Kinematic Fields: InfraRed Image Correlation (IRIC)," Experimental Mechanics, vol. 52, pp. 241-255, Mar. 2012.

[18] A. Charbal, J.-E. Dufour, A. Guery, F. Hild, S. Roux, L. Vincent, and M. Poncelet, "Integrated Digital Image Correlation considering gray level and blur variations: Application to distortion measurements of IR camera," Optics and Lasers in Engineering, vol. 78, pp. 75-85, Mar. 2016.

[19] A. Charbal, S. Roux, F. Hild, and L. Vincent, "Regularized infrared digital level corrections for digital image correlation," Quantitative InfraRed Thermography Journal, submitted, 2017.

[20] F. Hild, and S. Roux, "Digital image correlation: From measurement to identification of elastic properties - a review," Strain, vol. 42, pp. 69-80, 2006.

[21] S. Roux, and F. Hild, "Stress intensity factor measurements from digital image correlation: post-processing and integrated approaches," Int. J. Fract., vol. 140, no. 1-4, pp. 141-157, 2006.

[22] H. Leclerc, J.-N. Périé, S. Roux, and F. Hild, "Integrated Digital Image Correlation for the Identification of Mechanical Properties," in Computer Vision/Computer Graphics CollaborationTechniques: 4th International Conference, MIRAGE 2009, Rocquencourt, France, May 4-6, 2009. Proceedings (A. Gagalowicz and W. Philips, eds.), pp. 161-171, Berlin, Heidelberg: Springer Berlin Heidelberg, 2009.

[23] J. Réthoré, "A fully integrated noise robust strategy for the identification of constitutive laws from digital images," International Journal for Numerical Methods in Engineering, vol. 84, no. 6, pp. 631-660, 2010.

[24] F. Mathieu, H. Leclerc, F. Hild, and S. Roux, "Estimation of elastoplastic parameters via weighted FEMU and integrated-DIC," Exp Mech., vol. 55(1), 105-119, 2015. 
[25] M. Bertin, F. Hild, S. Roux, F. Mathieu, H. Leclerc, and P. Aimedieu, "Integrated digital image correlation applied to elastoplastic identification in a biaxial experiment," The Journal of Strain Analysis for Engineering Design, vol. 51, pp. 118-131, Feb. 2016.

[26] A. P. Ruybalid, J. P. M. Hoefnagels, O. van der Sluis, and M. G. D. Geers, "Comparison of the identification performance of conventional FEM updating and integrated DIC," International Journal for Numerical Methods in Engineering, vol. 106, no. 4, pp. 298-320, 2016.

[27] G. Besnard, S. Guérard, S. Roux, and F. Hild, "A space-time approach in digital image correlation: movie-DIC," Optics Lasers Engineering, vol. 49, pp. 71-81, 2011.

[28] G. Besnard, H. Leclerc, S. Roux, and F. Hild, "Analysis of image series through digital image correlation," Journal of Strain Analysis for Engineering Design, vol. 47, no. 4, pp. 214-228, 2012.

[29] G. B. Broggiato, L. Casarotto, Z. Del Prete, and D. Maccarrone, "Full-field strain rate measurement by white-light speckle image correlation," Strain, vol. 45 , no. 4 , pp. 364-372, 2009.

[30] J. Neggers, J. P. M. Hoefnagels, M. G. D. Geers, F. Hild, and S. Roux, "Time-resolved integrated digital image correlation," International Journal for Numerical Methods in Engineering, vol. 103, no. 3, pp. 157-182, 2015.

[31] A. Charbal, L. Vincent, F. Hild, M. Poncelet, J.-E. Dufour, S. Roux, and D. Farcage, "Characterization of temperature and strain fields during cyclic laser shocks," Quantitative InfraRed Thermography Journal, vol. 13, no. 1, pp. 1-18, 2016.

[32] K. Karhunen, "Über lineare Methoden in der Wahrscheinlichkeitsrechnung," Ann. Acad. Sci. Fennicae. Ser. A. I. Math.-Phys., vol. 37, p. 1979, 1947.

[33] M. Loève, Probability theory. Springer-Verlag, 1978.

[34] B. Pan, A. Asundi, H. Xie, and J. Gao, "Digital image correlation using iterative least squares and pointwise least squares for displacement field and strain field measurements," Optics and Lasers in Engineering, vol. 47, no. 7, pp. 865-874, 2009.

[35] B. Pan, H. M. Xie, and Z. Y. Wang, "Equivalence of Digital Image Correlation Criteria for Pattern Matching," Applied Optics, vol. 49, no. 28, pp. 5501-5509, 2010.

[36] L. Vincent, M. Poncelet, S. Roux, F. Hild, and D. Farcage, "Experimental Facility for High Cycle Thermal Fatigue Tests Using Laser Shocks," Procedia Engineering, vol. 66, pp. 669-675, 2013.

[37] A. Charbal, Mesure de champs thermomécaniques pour l'étude de la fatigue par chocs thermiques. PhD thesis, Université Paris-Saclay, 2017. 
[38] A. Charbal, J.-E. Dufour, F. Hild, M. Poncelet, L. Vincent, and S. Roux, "Hybrid stereocorrelation using infrared and visible light cameras," Experimental Mechanics, vol. 56, no. 5, 845-860, 2016.

[39] J. Chaboche, "Constitutive equations for cyclic plasticity and cyclic viscoplasticity," International Journal of Plasticity, vol. 5, pp. 247-302, Jan. 1989.

[40] P. Leplay, O. Lafforgue, and F. Hild, "Analysis of Asymmetrical Creep of a Ceramic at $1350{ }^{\circ} \mathrm{C}$ by Digital Image Correlation," Journal of the American Ceramics Society, vol. 98, no. 7, pp. 2240-2247, 2015. 